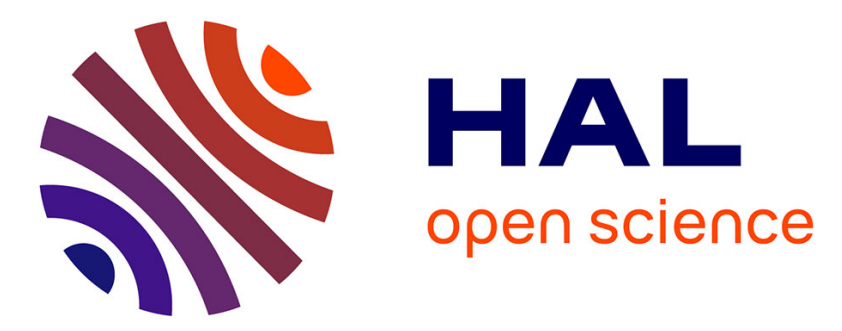

\title{
ANALYTIC AND GEOMETRIC PROPERTIES OF DISLOCATION SINGULARITIES
}

\author{
Riccardo Scala, Nicolas van Goethem
}

\section{To cite this version:}

Riccardo Scala, Nicolas van Goethem. ANALYTIC AND GEOMETRIC PROPERTIES OF DISLOCATION SINGULARITIES. 2016. hal-01297917

\section{HAL Id: hal-01297917 https://hal.science/hal-01297917}

Preprint submitted on 5 Apr 2016

HAL is a multi-disciplinary open access archive for the deposit and dissemination of scientific research documents, whether they are published or not. The documents may come from teaching and research institutions in France or abroad, or from public or private research centers.
L'archive ouverte pluridisciplinaire HAL, est destinée au dépôt et à la diffusion de documents scientifiques de niveau recherche, publiés ou non, émanant des établissements d'enseignement et de recherche français ou étrangers, des laboratoires publics ou privés. 
March 23, 2016

\title{
ANALYTIC AND GEOMETRIC PROPERTIES OF DISLOCATION SINGULARITIES
}

\author{
RICCARDO SCALA AND NICOLAS VAN GOETHEM
}

\begin{abstract}
This paper deals with the analysis of the singularities arising from the solutions of the problem Curl $F=\mu$, where $F$ is a $3 \times 3$ matrix-valued $L^{p}$-function $(1 \leq p<2)$ and $\mu$ a $3 \times 3$ matrix-valued Radon measure concentrated in a closed loop in $\Omega \subset \mathbb{R}^{3}$, or in a network of such loops, i.e., similar to dislocation clusters as observed in single crystals. In particular, we study the topological nature of such dislocation singularities. It is shown that $F=\nabla u$, the absolutely continuous part of the distributional gradient $D u$ of a vector-valued function $u$ of special bounded variation. Furthermore, $u$ can be redefined with values in the three-dimensional torus $\mathbb{T}^{3}$ and hence is Sobolev-regular away from the singular loops. We then analyze the graphs of such maps represented as currents on $\Omega \times \mathbb{T}^{3}$ and show that their boundaries can be written in term of the measure $\mu$. Readapting some well-known results for Cartesian currents, we recover closure and compactness properties of the class of maps with bounded curl concentrated on dislocation networks. We finally give some examples of variational problems where such results provide existence of solutions.
\end{abstract}

Key words: Cartesian maps, integer-multiplicity currents, torus-valued maps, dislocation singularity, variational problem.

AMS subject classification: 58A25, 46E40, 32C30, 49Q15, 53C65, 49J45, 74G65

\section{Contents}

1. Introduction

1.1. Line-like singularities Continuum mechanics

1.2. Scope and structure of the work

2. Preliminary results

2.1. Generalities about currents and graphs

2.2. Torus-valued maps and currents

2.3. Invertibility of the curl

3. Harmonic maps with prescribed jump on a surface

3.1. Pointwise and distributional properties of gradients of harmonic maps

3.2. Distributional properties

4. Main result: graph boundary of $\mathbb{T}^{3}$-valued harmonic maps 16

4.1. Statement of the main theorem $r$

4.2. Extensions of Theorem $4.1 \quad 19$

5. Main result second form: Euclidean setting and jump surface 21

5.1. Expression of the boundary graph for $\mathbb{R}^{3}$-valued displacement 21

6. Application to dislocations at the continuum scale 23

6.1. Preliminaries on dislocations at the continuum scale 23

6.2. Functional properties $\quad 24$

6.3. Expression of Theorem 4.1 for a finite number of dislocation loops 24

6.4. Two examples 26

6.5. Physical motivations of the Euclidean-valued case $r$

$\begin{array}{llr}\text { Appendix A. } & \text { Proof of Theorem 2.6 } & 29\end{array}$

$\begin{array}{lll}\text { Appendix B. } & \text { Proof of Theorem 5.1 } & 31\end{array}$

$\begin{array}{ll}\text { Acknowledgements } & 32\end{array}$

$\begin{array}{lr}\text { References } & 32\end{array}$ 


\section{INTRODUCTION}

In this work we analyze the class of $3 \times 3$ matrices-valued fields $F$ on an a open set $\Omega \subset \mathbb{R}^{3}$ which have singularities on a 1-dimensional set $\mathcal{L}$. The singularity we are interested in arises from the condition that $F$ is assumed to have a nonzero Curl, which is a finite Radon measure $\mu$ concentrated on $\mathcal{L}$, namely

$$
-\operatorname{Curl} F=\mu \text {. }
$$

In the simplest case when the singular set is a closed Lipschitz curve $\mathcal{L}$, then it is well-known that $\mu$ must be a so called dislocation density, that is a measure of the form

$$
\mu=b \otimes \tau \mathcal{H}_{\llcorner\mathcal{L}}^{1},
$$

where $b$ is a fixed vector (constant along $\mathcal{L}$ and called Burgers vector) and $\tau$ is the unit tangent vector to $\mathcal{L}$ (see Lemma 6.1). The term "dislocation" comes from the physics of crystals, where the strain field of the elastic body cannot be in general associated to a global deformation, since it is observed to satisfy a condition like (1.1). In dislocation theory, the singular set $\mathcal{L}$ is always a countable (or finite) union of Lipschitz closed curves, and hence the measure $\mu$ is a sum of countable many dislocation densities of the form (1.2). Each Burgers vector of the singletons (1.2) is constrained to belong to a countable lattice, that we assume to be $\mathbb{Z}^{3}$. This setting can be summarized by asking that the measure $\mu$ in (1.1) is representable by a closed integral current with coefficients in the group $\mathbb{Z}^{3}$.

By Helmholtz decomposition, any tensor-valued $F \in L^{p}(\Omega)$ can be written as $F=D u+$ Curl $V$, with $u \in W^{1, p}(\Omega)$ and $V$ a divergence-free tensor, see [22]. It is a standard interpretation to call $u$ the displacement field, and $V$ the incompatible field. However it is immediately observed by (1.1) that the dislocation measure $\mu$ depends on $V$ only, hence leaving the displacement outside the picture, at least as far as classically intended as a Sobolev vector-valued map.

The class of vector fields $F: \Omega \rightarrow \mathbb{R}^{3}$ satisfying condition (1.1) with $\mu$ a closed integral current with coefficients in $\mathbb{Z}^{3}$ has several measure theoretic properties that are proved in the present paper. A central aspect is that for every $F$ there exists a 3 -dimensional torus valued map $u: \Omega \rightarrow \mathbb{T}^{3}$ such that $F=\nabla u$. In some sense, even if the non-vanishing of Curl $F$ precludes the existence of a vector-valued function whose gradient is $F$, we recover such property in the class of torus-valued vector fields. As a consequence, in the spirit of [18], the graph of the map $u$ can be seen as a current and a theory as for Cartesian Currents can be developed. Our main result gives the explicit form of the boundary of the graph associated to the torus-valued deformation.

1.1. Line-like singularities Continuum mechanics. A dislocation (or disclination) is a line defect in a crystal related to the jump of the displacement or the rotation tensor, itself provoked by the motion of atoms and atom layers at an underlying microscopic scale. At the mesoscopic scale, where the medium is considered as continuous, such a line represents a singularity for model fields such as stress, strain, displacement and rotation. Furthermore, in single crystals these lines might appear as isolated loops as well as complex networks and there are such interactions that two lines might join together or (partially) annihilate, or be set apart in many smaller loops. All these phenomena should be understood as mathematical objects which must be described in a geometrically unified way. To achieve this goal, the theory of currents has shown as extremely appropriate, as described in [29]. It should be emphasized that integral currents and Cartesian currents also allow one to handle singular problems in Continuum mechanics by means of the notions of distributional Jacobian and cofactors of the deformation tensor. Pioneer works and fundamental results about this topic can be found in [26]. In the recent years, this formalism is being developed for elasticity and in general for Continuum mechanics, as in, e.g., $[2,3,16,19,24,25]$. About their specific use in problems involving dislocations, see $[27,29]$. About their use in problems involving other kinds of loop discontinuities, the Ginzburg and Landau problem has provided numerous key contributions, as e.g. in [1,6,28]. Indeed, the mathematical issues addressed in these works very often appear in a similar way in the study of defects in solids, which therefore shares with Ginzburg-Landau models several theoretical concepts, 
among which field multiple-valuedness and vorticity. Let us also mention function of higher-order variation [20] (considered in [29] for dislocation problems), or strings [9], as one of the many mathematical objects arisen from the study of singular loops in PDEs. It should be stressed that one of the feature of these problems is that the model fields have either manifold-valued (for instance, in the unit sphere), or are multiple valued. Multivaluedness is a challenging issue since the pioneer works of Almgren [4], both in Physics problems such as liquid crystals, or from a purely mathematical perspective $[7,12]$, where often, multivaluedness is related to singularities, as in [10].

Sometimes, dislocations are considered in a unified way with fracture, as e.g. in [24], where "fracture" is intended as a non-negative co-dimension singularity, i.e., classical fracture have codimension 1, whereas dislocations have co-dimension 2, and point defects co-dimension 3. However, dislocation singularities show several specific issues, and the intrinsic mathematical difficulties generated by dislocations are fundamentally different from those encountered in the mathematical modeling of fracture mechanics. Indeed,

(i) dislocations are $\mathcal{H}^{1}$-singularities in $\mathbb{R}^{3}$ and hence show no jump set in the classical sense;

(ii) dislocations are free to mutually annihilate, recombine, split, spontaneously appear, and hence form complex geometrical structures, without any law such as irreversibility;

(iii) there is no natural reference configuration and hence intrinsic approaches must be preferred. In particular, the displacement is not an appropriate model variable in the classical sense, as opposed to most of Solid Mechanics problems;

(iv) the stress and strain fields are not square-integrable and so the less tractable $L^{p}$ spaces with $1 \leq p<2$ must be considered;

(v) bounds on the model fields are given in terms of the curl and the divergence, in place of the full gradient;

(vi) these curl and divergence are found in measure spaces instead of Sobolev spaces.

In the language of currents, the measure (1.2) is denoted as $b \otimes \mathcal{L}$, with $\mathcal{L}$ standing for an integral 1-current, as described in [29]. In the language of Physics, it is simply the (transpose of) the dislocation density $\Lambda_{\mathcal{L}}:=\tau \otimes b \mathcal{H}_{\llcorner\mathcal{L}}^{1}$, which should be considered as the problem data. The tensor-valued functions satisfying the differential constraint (1.1) will be denoted by $F$ to mean the deformation tensor in finite elasticity. Because of this constraint, $F$ belongs to $L^{p}(\Omega)$ with $1 \leq p<2$. It is a classical procedure to also consider the determinant and cofactors of $F$ as belonging to the same space, since J. Ball results on existence results for polyconvex functions $[5,11]$. Such an issue will be addressed in a forthcoming work, as a natural consequence of the analysis of the present paper.

1.2. Scope and structure of the work. As a first step, our aim is to analyze the fields satisfying the constraint (1.1), showing that an unambiguous concept of displacement can be defined. As a second step, we want to study some measure theoretic properties of such displacements, showing some important closure results. More specifically, it will be shown that

- For all $F$ satisfying (1.1) there exists a map $u \in W^{1, p}\left(\Omega, \mathbb{T}^{3}\right)$, with $\mathbb{T}=\mathbb{R} / \mathbb{Z}$ the flat torus, such that $F=\nabla u$. Moreover there exists a map $\tilde{u} \in S B V^{p}\left(\Omega, \mathbb{R}^{3}\right)$ such that $F=\nabla^{a} \tilde{u}$, with $\nabla^{a} \tilde{u}$ the absolutely continuous part of the distributional gradient $D \tilde{u}$.

- $u \in W^{1, p}\left(\Omega, \mathbb{T}^{3}\right)$ can always be decomposed as $u=v+w$, with $v \in W^{1, p}\left(\Omega, \mathbb{T}^{3}\right)$ harmonic in $\Omega \backslash \mathcal{L}$, and where $w \in W^{1, p}\left(\Omega, \mathbb{R}^{3}\right)$ is vector-valued. Moreover $v \in \mathcal{C}^{\infty}\left(\Omega \backslash \mathcal{L}, \mathbb{T}^{3}\right)$, that is, $u$ is smooth except on the singularity $\mathcal{L}$.

- $\partial \mathcal{G}_{v}$, the boundary of the current supported by the graph of $v$, is "equals" (in a sense that will be made precise) to $\mu=-\operatorname{Curl}^{T} \nabla u$.

The first item states that it is possible to construct a real-valued displacement field $\tilde{u}$, which has a constant jump $b$ on a fixed (though arbitrary) surface $S$ enclosed by $C$. If we quotient the target space $\mathbb{R}^{3}$ by $\mathbb{Z}^{3}$, the jump on the (arbitrary) surface is neglected. In other words, the displacement as an Euclidean-valued field is multiple-valued, since its circulation around dislocation lines is nonzero, and is 
always a multiple of $b$. Otherwise, working with torus-valued maps allows us to remove this ambiguity. Torus-valued maps are therefore alternatives to Euclidean maps appropriate to mathematically model dislocation singularities. Similarly, it will be convenient to introduce periodic $k$-forms, from which torus-valued currents are defined by duality, the latter being more suitable in this setting. Note that such multivalued displacement fields have been used by Physicists, for a long time (see, e.g., [21]), but without the necessary mathematical rigor, which we believe is worth introducing to seek a profound understanding of the intrinsic difficulties generated by dislocation singularities.

As for the last item, with the language of currents and in dislocation terms, our main result basically states that the dislocation density is the boundary of the graph of a displacement field $u$ which has values in the three dimensional torus, viz.,

$$
\partial \mathcal{G}_{u}(\omega)=\mathcal{L} \otimes b\left(\varphi^{\omega}\right),
$$

where $\omega$ is any $n$-form, and $\varphi^{\omega}$ is a test function depending linearly on $\omega$ (see Theorem 6.3 for details). Starting from this result it is possible to write the explicit form of the graph boundary of $w=v+u$, a smooth perturbation $v$ of the function $u$. Finally, we give the explicit form of the graph boundary of the vector-valued function $\tilde{u}$, which indeed coincides with the torus-valued one $u$, with a additional measure concentrated on the jump set of $\tilde{u}$ (see Theorem 5.1 for details); this is our main result second form. It might be used in models involving an energy term on the jump surface, whose complete analysis, though, is left for future work.

Finally, application examples are given. In particular, two variational problems are proposed and solved in Section 6.4.

This paper is structured as follows. In Section 2, we recall the basis for currents and the theory of graphs of torus-valued maps, and prove some important preliminary results, whose counterparts in the Euclidean-valued case are classical. Then, the crucial pointwise and distributional properties of torus-valued harmonic maps are derived in Sections 3. The main results are presented in two forms, the first in Section 4 holding for torus-valued maps (see Theorem 4.1), and the second one valid for Euclidean maps is given in Section 5, with a proof in Appendix B. In Section 6 we show how the results obtained so far can be applied to the theory of dislocations, whereas in Section 6.4 we provide two examples of variational problems which can be solved thanks to our main results and their applications.

\section{Preliminary Results}

2.1. Generalities about currents and graphs. For all integers $n>0$ and $k \geq 0$ with $k \leq n$, we denote by $\Lambda_{k} \mathbb{R}^{n}$ the space of $k$-vectors and by $\Lambda^{k} \mathbb{R}^{n}$ the space of $k$ covectors. Let $\alpha$ be a multi-index, i.e., an ordered (increasing) subset of $\{1,2, \ldots, n\}$. We denote by $|\alpha|$ the cardinality (or length) of $\alpha$, and we denote by $\bar{\alpha}$ the complementary set of $\alpha$, i.e., the multi-index given by the ordered set $\{1,2, \ldots, n\} \backslash \alpha$.

For a $n \times n$ matrix $A$ with real entries and for $\alpha$ and $\beta$ multi-indexes such that $|\alpha|+|\beta|=n$, $M_{\bar{\alpha}}^{\beta}(A)$ will denote the determinant of the submatrix of $A$ given by erasing the $i$-th columns and the $j$-th rows, for all $i \in \alpha$ and $j \in \bar{\beta}$. Moreover, symbol $M(A)$ will denote the $n$-vector in $\Lambda_{n} \mathbb{R}^{2 n}$ given by

$$
M(A):=\sum_{|\alpha|+|\beta|=n} \sigma(\alpha, \bar{\alpha}) M_{\bar{\alpha}}^{\beta}(A) e_{\alpha} \wedge \varepsilon_{\beta},
$$

where $\left\{e_{i}, \varepsilon_{i}\right\}_{i \leq n}$ is the Euclidean basis of $\mathbb{R}^{2 n}$, and $\sigma(\alpha, \bar{\alpha})$ is the sign of the permutation $(\alpha, \bar{\alpha})$. Accordingly, define

$$
|M(A)|:=\left(1+\sum_{\substack{|\alpha|+|\beta|=n \\|\beta|>0}}\left|M_{\bar{\alpha}}^{\beta}(A)\right|^{2}\right)^{1 / 2} .
$$


For a matrix $A \in \mathbb{R}^{3 \times 3}$, the symbols adj $A$ and $\operatorname{det} A$ denote the adjunct, i.e., the transpose of the matrix of the cofactors of $A$, and the determinant of $A$, respectively. Explicitly,

$$
M_{j}^{i}(A)=A_{i j}, \quad M_{\bar{j}}^{\bar{i}}(A)=(\operatorname{cof} A)_{i j}=(\operatorname{adj} A)_{j i} \quad M_{\{1,2,3\}}^{\{1,2,3\}}(A)=\operatorname{det} A,
$$

for $i, j=1,2,3$.

Moreover,

$$
|M(A)|=\left(1+\sum_{i, j} A_{i j}^{2}+\sum_{i, j} \operatorname{cof}(A)_{i j}^{2}+\operatorname{det}(A)^{2}\right)^{1 / 2}
$$

Let $\Omega \subset \mathbb{R}^{n}$ be an open set, then $\mathcal{D}^{k}(\Omega)$ denotes the topological vector space of compactly supported smooth $k$-forms on $\Omega$, that is the topological vector space of compactly supported and smooth maps on $\Omega$ with values in $\Lambda^{k} \mathbb{R}^{n}$. The dual space of $\mathcal{D}^{k}(\Omega)$, denoted by $\mathcal{D}_{k}(\Omega)$, is known as the space of $k$-currents on $\Omega$. A weak convergence in $\mathcal{D}_{k}(\Omega)$ is defined by saying that $\mathcal{T}_{h} \rightarrow \mathcal{T}$ as currents if for all $\omega \in \mathcal{D}^{k}(\Omega)$ we have $\mathcal{T}_{h}(\omega) \rightarrow \mathcal{T}(\omega)$. The boundary of a current $\mathcal{T} \in \mathcal{D}_{k}(\Omega)$ is the current $\partial \mathcal{T} \in \mathcal{D}_{k-1}(\Omega)$ defined as

$$
\partial \mathcal{T}(\omega)=\mathcal{T}(d \omega), \quad \text { for all } \omega \in \mathcal{D}^{k-1}(\Omega) .
$$

A current which has null boundary (as any current in $\mathcal{D}_{0}(\Omega)$ ) is said closed. For all $\mathcal{T} \in \mathcal{D}_{k}(\Omega)$ the mass of $\mathcal{T}$ is the number $M(\mathcal{T}) \in[0,+\infty]$ defined as

$$
M(\mathcal{T}):=\sup _{\omega \in \mathcal{D}^{k}(\Omega),|\omega| \leq 1} \mathcal{T}(\omega) .
$$

If $M(\mathcal{T})<+\infty$ then $\mathcal{T}$ turns out to be a Borel measure in $\mathcal{M}_{b}\left(\Omega, \Lambda_{k} \mathbb{R}^{n}\right)$, and its mass coincides with $M(\mathcal{T})$. Moreover the mass is lower semicontinuous with respect to the weak topology in $\mathcal{D}_{k}(\Omega)$. Indeed if $\limsup _{h \rightarrow \infty} M\left(\mathcal{T}_{h}\right)<+\infty$ and $\mathcal{T}_{h} \rightarrow \mathcal{T}$ then we also find that $\mathcal{T}$ is a Borel measure and $\mathcal{T}_{h} \rightarrow \mathcal{T}$ weakly in $\mathcal{M}_{b}\left(\Omega, \Lambda^{k} \mathbb{R}^{n}\right)$, so that the lower-semicontinuity of the mass follows from the lower-semicontinuity of the mass in $\mathcal{M}_{b}\left(\Omega, \Lambda^{k} \mathbb{R}^{n}\right)$. We also define the quantity

$$
N(\mathcal{T}):=M(\mathcal{T})+M(\partial \mathcal{T})
$$

for every $\mathcal{T} \in \mathcal{D}_{k}(\Omega)$

Let $U \subset \mathbb{R}^{n}$ and $V \subset \mathbb{R}^{m}$ be open sets and $F: U \rightarrow V$ be a smooth map. Then the push-forward of a current $\mathcal{T} \in \mathcal{D}_{k}(U)$ by $F$ is defined as

$$
F_{\sharp} \mathcal{T}(\omega):=\mathcal{T}\left(\zeta F^{\sharp} \omega\right) \quad \text { for } \omega \in \mathcal{D}^{k}(V),
$$

where $F^{\sharp} \omega$ is the standard pull-back of $\omega$ and $\zeta$ is any $\mathcal{C}^{\infty}$ function that is equal to 1 on $\operatorname{spt} \mathcal{T} \cap \operatorname{spt} F^{\sharp} \omega$. It turns out that $F_{\sharp} \mathcal{T} \in \mathcal{D}_{k}(V)$ does not depend on $\zeta$ and satisfies

$$
\partial F_{\sharp} \mathcal{T}=F_{\sharp} \partial \mathcal{T} \text {. }
$$

Let $0 \leq k \leq n$ and let $S \subset \mathbb{R}^{n}$ be $\mathcal{H}^{k}$-rectifiable set with approximate tangent space $T_{x} S$. If $\tau: S \rightarrow \Lambda_{k}\left(\mathbb{R}^{n}\right)$ and $\theta: S \rightarrow \mathbb{R}$ are $\mathcal{H}^{k}$-integrable functions with $\tau(x) \in T_{x} S$ a simple unit $k$-vector for $\mathcal{H}^{k}$-a.e. $x \in S$, then we can define the current $\mathcal{T}$ as

$$
\mathcal{T}(\omega)=\int_{S}\langle\omega(x), \tau(x)\rangle \theta(x) d \mathcal{H}^{k}(x) \quad \text { for } \omega \in \mathcal{D}^{k}(\Omega) .
$$

Every current for which there exists $S, \tau$, and $\theta$ as before is said to be rectifiable. If also its boundary $\partial \mathcal{T}$ is rectifiable, then we adopt the following notation

$$
\mathcal{T} \equiv\{S, \tau, \theta\}
$$

The current $\mathcal{T} \in \mathcal{D}_{k}(\Omega)$ is rectifiable with integer multiplicity if it is rectifiable, has rectifiable boundary, and the function $\theta$ in (2.3) is integer valued. A integer-multiplicity current $\mathcal{T}$ such that $N(\mathcal{T})<\infty$ is said integral current. 
An integer-multiplicity current $\mathcal{T} \in \mathcal{D}_{M}\left(\mathbb{R}^{n}\right)$ is said indecomposable if there exists no integral current $\mathcal{R}$ such that $\mathcal{R} \neq 0 \neq \mathcal{T}-\mathcal{R}$ and

$$
N(\mathcal{T})=N(\mathcal{R})+N(\mathcal{T}-\mathcal{R})
$$

The following theorem provides the decomposition of every integral current and the structure of integer-multiplicity indecomposable 1-current (see [13, Section 4.2.25]).

Theorem 2.1. For every integer multiplicity current $\mathcal{T}$ there exists a sequence of indecomposable integral currents $\mathcal{T}_{i}$ such that

$$
\mathcal{T}=\sum_{i} \mathcal{T}_{i} \quad \text { and } \quad N(\mathcal{T})=\sum_{i} N\left(\mathcal{T}_{i}\right)
$$

Suppose $\mathcal{T}$ is an indecomposable integer multiplicity 1 -current on $\mathbb{R}^{n}$. Then there exists a Lipschitz function $: \mathbb{R} \rightarrow \mathbb{R}^{n}$ with $\operatorname{Lip}(f) \leq 1$ such that

$$
f\left\llcorner[0, M(\mathcal{T})] \text { is injective and } \mathcal{T}=f_{\sharp}[0, M(\mathcal{T})]\right. \text {. }
$$

Moreover $\partial \mathcal{T}=0$ if and only if $f(0)=f(M(\mathcal{T}))$.

Let us consider the space $\Omega \times \mathbb{R}^{3}$. We will use the Euclidean coordinates $x=\left(x_{1}, x_{2}, x_{3}\right)$ for $x \in \Omega$ and $y=\left(y_{1}, y_{2}, y_{3}\right)$ for $y \in \mathbb{R}^{3}$. Every 3-form $\omega \in \mathcal{D}^{3}\left(\Omega \times \mathbb{R}^{3}\right)$ can be decomposed as $\omega=\sum \omega_{\alpha \beta} d x_{\alpha} \wedge d y_{\beta}$, with $\omega_{\alpha \beta} \in C_{c}^{\infty}\left(\Omega \times \mathbb{R}^{3}\right)$, and where the sum is computed over all multi-indexes $\alpha$ and $\beta$ such that $|\alpha|+|\beta|=3$.

For $1 \leq p<+\infty$ we define

$$
\begin{gathered}
\mathcal{A}_{p}(\Omega):=\left\{u \in L^{p}\left(\Omega, \mathbb{R}^{3}\right): u\right. \text { is approx. differentiable a.e. and } \\
\left.M_{\bar{\alpha}}^{\beta}(D u) \in L^{p}(\Omega) \text { for all }|\alpha|+|\beta|=3\right\} .
\end{gathered}
$$

A weak convergence is defined on $\mathcal{A}_{p}(\Omega)$. We say that the sequence $u_{h} \in \mathcal{A}_{p}(\Omega)$ converges to $u \in \mathcal{A}_{p}(\Omega)$ weakly in $\mathcal{A}_{p}(\Omega)$ if $u_{h} \rightarrow u$ weakly in $L^{p}\left(\Omega, \mathbb{R}^{3}\right)$ and $M_{\bar{\alpha}}^{\beta}\left(D u_{h}\right) \rightarrow M_{\bar{\alpha}}^{\beta}(D u)$ weakly in $L^{p}(\Omega)$ for all multi-indexes $\alpha$ and $\beta$ with $|\alpha|+|\beta|=3$ (see [18, Section 3.3.3]). If $u \in \mathcal{A}_{p}(\Omega)$ then $\mathcal{G}_{u}$, the current carried by the graph of $u$, is well defined as follows:

$$
\mathcal{G}_{u}:=(I d \times u)_{\sharp} \llbracket \Omega \rrbracket,
$$

where $I d \times u: \mathbb{R}^{3} \rightarrow \mathbb{R}^{3} \times \mathbb{R}^{3}$ is given by $(I d \times u)(x)=(x, u(x))$, viz.,

$$
\mathcal{G}_{u}(\omega)=\int_{\Omega} \sigma(\alpha, \bar{\alpha}) \omega_{\alpha \beta}(x, u(x)) M_{\bar{\alpha}}^{\beta}(D u(x)) d x,
$$

for all $\omega=\omega_{\alpha \beta} d x_{\alpha} \wedge d y_{\beta} \in \mathcal{D}^{3}\left(\Omega \times \mathbb{R}^{3}\right)$. Moreover if $u \in \mathcal{A}_{p}(\Omega)$ then $\mathcal{G}_{u}$ turns out to be a 3 -integermultiplicity current in $\Omega \times \mathbb{R}^{3}$.

Lemma 2.2. Let $u_{\epsilon}, u \in \mathcal{A}_{p}(\Omega)$ be such that $u_{\epsilon} \rightarrow u$ weakly in $\mathcal{A}_{p}(\Omega)$, then $\mathcal{G}_{u_{\epsilon}} \rightarrow \mathcal{G}_{u}$ as currents.

Proof. This is a straightforward consequence of formula (2.6).

The boundary of the current $\mathcal{G}_{u}(\omega)$ is, by definition, the 2 -current in $\Omega \times \mathbb{R}^{3}$ given by $\partial \mathcal{G}_{u}(\omega):=$ $\mathcal{G}_{u}(d \omega)$ for all 2 -forms $\omega \in \mathcal{D}^{2}\left(\Omega \times \mathbb{R}^{3}\right)$. As soon as $u\llcorner U$, the restriction of $u$ to a smooth open subset $U$ of $\Omega$, is assumed smooth in $U$, then $\mathcal{G}_{u\llcorner U}$ is a surface with rectifiable boundary given by

$$
\partial \mathcal{G}_{u\llcorner U}=\mathcal{G}_{u\llcorner\partial U}:=(I d \times u)_{\sharp} \llbracket \partial U \rrbracket .
$$

We can also employ Stokes formula to find an explicit formula for $\partial \mathcal{G}_{u\llcorner\partial U}$, i.e.,

$$
\mathcal{G}_{u\llcorner U}(d \omega)=\partial \mathcal{G}_{u\llcorner U}(\omega)=\int_{\partial U} \sigma(\alpha, \bar{\alpha}) \omega_{\alpha \beta}(u, u(x)) M_{\bar{\alpha}}^{\beta}\left(D u\llcorner\partial U)(x) d \mathcal{H}^{2}(x),\right.
$$

for all 2-form $\omega=\omega_{\alpha \beta} d x_{\alpha} \wedge d y_{\beta} \in \mathcal{D}^{2}\left(\Omega \times \mathbb{R}^{3}\right)$. This can be seen as follows: by definition of pushforward of a current, $\mathcal{G}_{u\llcorner\partial U}(\omega)=\llbracket \partial U \rrbracket\left((I d \times u)^{\sharp}(\omega)\right)=\int_{\partial U}\left\langle(I d \times u)^{\sharp} \omega, \tau^{1} \wedge \tau^{2}\right\rangle d \mathcal{H}^{2}=\int_{\partial U}\left\langle\omega, \frac{\partial \Psi}{\partial \tau^{1}} \wedge\right.$ 
$\left.\frac{\partial \Psi}{\partial \tau^{2}}\right\rangle d \mathcal{H}^{2}$ where $\tau^{1} \wedge \tau^{2}$ is a volume form for $\partial U$. Now by Stokes Theorem this is equal to (recall that one can interchange the exterior differentiation and pull-back operations) $\int_{U}\left\langle d(I d \times u)^{\sharp} \omega, e^{1} \wedge e^{2} \wedge e^{3}\right\rangle d \mathcal{H}^{3}=$ $\int_{U}\left\langle(I d \times u)^{\sharp} d \omega, e^{1} \wedge e^{2} \wedge e^{3}\right\rangle d \mathcal{H}^{3}=\mathcal{G}_{u\llcorner U}(d \omega)=\partial \mathcal{G}_{u\llcorner U}(\omega)$ (see [17] p.272 or [23] for details).

The class of Cartesian maps is the subspace of $\mathcal{A}_{1}\left(\Omega, \mathbb{R}^{3}\right)$ defined as

$$
\operatorname{Cart}^{1}\left(\Omega, \mathbb{R}^{3}\right):=\left\{u \in \mathcal{A}_{1}\left(\Omega, \mathbb{R}^{3}\right): \partial \mathcal{G}_{u}=0\right\} .
$$

If $u \in W^{1, p}\left(\Omega, \mathbb{R}^{3}\right)$ with $p>3$, then it is easy to see that $u \in \operatorname{Cart}^{1}\left(\Omega, \mathbb{R}^{3}\right)$. See $[18$, Section 3.2.2] for details.

Theorem 2.3. Let $p>1$. Let $u_{k}$ be a sequence in $\mathcal{A}_{p}\left(\Omega, \mathbb{R}^{3}\right)$ such that $u_{k} \rightarrow u$ strongly in $L^{p}\left(\Omega, \mathbb{T}^{3}\right)$ and suppose that there exist functions $v_{\beta}^{\alpha} \in L^{p}(\Omega)$ such that $M_{\bar{\alpha}}^{\beta}\left(D u_{k}\right) \rightarrow v_{\alpha}^{\beta}$ for all multi-indexes $\alpha$ and $\beta$ with $|\alpha|+|\beta|=3$. If

$$
M\left(\partial \mathcal{G}_{u_{k}}\right)<C<+\infty
$$

for all $k>0$, then $u \in \mathcal{A}_{p}\left(\Omega, \mathbb{R}^{3}\right)$ and $v_{\alpha}^{\beta}=M_{\bar{\alpha}}^{\beta}(D u)$.

This is proved in Theorem 2 of [18, Section 3.3.2]. Since Cartesian maps obviously satisfy condition (2.9), this Theorem applies to this case. Moreover if $u_{k} \in \operatorname{Cart}^{1}\left(\Omega, \mathbb{R}^{3}\right)$ then we also have $u \in \operatorname{Cart}^{1}\left(\Omega, \mathbb{R}^{3}\right)$.

2.2. Torus-valued maps and currents. For simplicity of exposition, we describes the three dimensional case, but all the definitions and statements of this section can be established for the general $n$-dimensional case. We introduce the torus $\mathbb{T} \cong \mathbb{R} / \sim$, where $\sim$ denotes the equivalent relation given by $a \sim b$ iff $a-b \in 2 \pi \mathbb{Z}$. We also denote $\mathbb{T} \times \mathbb{T} \times \mathbb{T}$ by $\mathbb{T}^{3}$. Now we will consider graphs of maps $u: \Omega \rightarrow \mathbb{T}^{3}$. These turn out to be 3-rectifiable currents in $\Omega \times \mathbb{T}^{3}$. Note that the space of 3 -forms in $\Omega \times \mathbb{T}^{3}$, i.e. $\mathcal{D}^{3}\left(\Omega \times \mathbb{T}^{3}\right)$, is exactly the space of 3 -forms in $\Omega \times \mathbb{R}^{3}$ that have coefficients which are smooth and $2 \pi$-periodic (with all their derivatives) in the last three variables (actually, they do not have compact support). As a consequence, if $\mathcal{T}$ is a 3 -current in $\mathcal{D}_{3}\left(\Omega \times \mathbb{R}^{3}\right)$ that has compact support in $\bar{\Omega} \times \mathbb{R}^{3}$, then the current

$$
T(\mathcal{T}):=\mathcal{T}\left\llcorner_{\mathcal{D}^{3}\left(\Omega \times \mathbb{T}^{3}\right)},\right.
$$

is well-defined and $T(\mathcal{T}) \in \mathcal{D}_{3}\left(\Omega \times \mathbb{T}^{3}\right)$. Moreover, since in general smooth functions in $\Omega \times \mathbb{R}^{3}$ are not periodic in the last three variables, it turns out that $M(T(\mathcal{T})) \leq M(\mathcal{T})$.

Let $u \in \mathcal{A}_{p}\left(\Omega, \mathbb{R}^{3}\right)$, then we define $T(u): \Omega \rightarrow \mathbb{T}^{3}$ by means of the standard projection $\pi_{T}: \mathbb{R} \rightarrow \mathbb{T}$, i.e. $T\left(u_{i}\right):=\pi_{T}\left(u_{i}\right)$ for $i=1,2,3$. It is easily seen that, $\mathbb{T}$ being locally isomorphic to $\mathbb{R}$, $T(u)$ is almost everywhere approximately differentiable with the same approximate derivatives of $u$. As a consequence $\mathcal{G}_{T(u)}$ is a 3 -rectifiable current in $\Omega \times \mathbb{T}^{3}$. It is also easy to see that in such a case $\mathcal{G}_{T(u)}=T\left(\mathcal{G}_{u}\right)$. This fundamental identity follows from the fact that the approximate differentials of $u$ and $T(u)$ coincide almost everywhere, and from (2.6), where we use that if $\omega$ is $2 \pi$-periodic in the second variable, then $\omega(x, u(x))=\omega(x, T(u(x)))$. We introduce the space $\mathcal{A}_{p}\left(\Omega, \mathbb{T}^{3}\right)$ as follows:

\section{Definition 2.4.}

$$
\begin{aligned}
\mathcal{A}_{p}\left(\Omega, \mathbb{T}^{3}\right):= & \left\{u \in L^{1}\left(\Omega, \mathbb{T}^{3}\right): u \text { is approx. differentiable a.e. on } \Omega,\right. \text { and } \\
& \left.M_{\bar{\alpha}}^{\beta}(D u) \in L^{p}(\Omega) \text { for all }|\alpha|+|\beta|=3\right\},
\end{aligned}
$$

where $L^{1}\left(\Omega, \mathbb{T}^{3}\right)$ means the space of measurable functions $u: \Omega \rightarrow \mathbb{T}^{3}$.

With this definition, we see that for all $u \in \mathcal{A}_{p}\left(\Omega, \mathbb{T}^{3}\right)$ the graph $\mathcal{G}_{u}$ is well defined as a 3rectifiable current. A consequence of the fact that the mass of a current does not increase when we compose with $T$ is that, if there exists $\bar{u} \in \mathcal{A}_{p}\left(\Omega, \mathbb{R}^{3}\right)$ such that $T(\bar{u})=u$ and $\mathcal{G}_{\bar{u}}$ is an integral current, 
then $\mathcal{G}_{u}$ is an integral current. Note that it might happen that such $\bar{u}$ does exist with $\partial \mathcal{G}_{\bar{u}}$ unbounded, while $M\left(\partial \mathcal{G}_{u}\right)<\infty$.

Theorem 2.3, being a consequence of the compactness theorem for integral currents, straightforwardly applies also to the case of maps with values in $\mathbb{T}^{3}$.

Lemma 2.2 readily applies to the case of maps with value in $\mathbb{T}^{3}$.

Lemma 2.5. Let $u_{\epsilon}, u \in \mathcal{A}_{p}\left(\Omega, \mathbb{T}^{3}\right)$ be such that $u_{\epsilon} \rightarrow u$ weakly in $\mathcal{A}_{p}\left(\Omega, \mathbb{T}^{3}\right)$, then $\mathcal{G}_{u_{\epsilon}} \rightarrow \mathcal{G}_{u}$ as currents.

Proof. This is again a consequence of formula (2.6) and the fact that currents in $\mathcal{D}_{3}\left(\Omega, \mathbb{T}^{3}\right)$ belong also to $\mathcal{D}_{3}\left(\Omega, \mathbb{R}^{3}\right)$.

2.3. Invertibility of the curl. In this section we introduce a technical result that will be useful for the subsequent discussion. in $\mathbb{R}^{3 \times 3}$.

Let $\mathcal{M}_{\text {div }}\left(\hat{\Omega}, \mathbb{R}^{3 \times 3}\right)$ denote the space of divergence-free bounded Radon measures with values

Theorem 2.6 (Biot-Savart). Let $\hat{\Omega} \subset \mathbb{R}^{3}$ be an open simply connected and smooth set. Let $\mu$ be a tensor-valued Radon measure such that $\mu \in \mathcal{M}_{\operatorname{div}}\left(\hat{\Omega}, \mathbb{R}^{3 \times 3}\right)$. Then there exists a unique $F \in$ $L^{1}\left(\hat{\Omega}, \mathbb{R}^{3 \times 3}\right)$ solution of

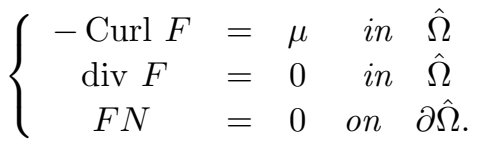

Moreover $F$ belongs to $L^{p}\left(\hat{\Omega}, \mathbb{R}^{3 \times 3}\right)$ for all $p$ with $1 \leq p<3 / 2$ and for all such $p$ there exists a constant $C>0$ satisfying

$$
\|F\|_{p} \leq C|\mu|(\hat{\Omega}) .
$$

In the case that $\mu=\tau \otimes b \mathcal{H}^{1}\left\llcorner_{L}\right.$, for some $b \in \mathbb{R}^{3}$ and $a \mathcal{C}^{2}$-closed curve $L$ in $\hat{\Omega}$ with unit oriented tangent vector $\tau$, then the solution $F$ belongs to $L^{p}\left(\hat{\Omega}, \mathbb{R}^{3 \times 3}\right)$ for all $p<2$.

The proof is a straightforward extensions of the main results in [8], and thus we discuss it in the appendix.

\section{HARMONIC MAPS WiTh PRESCRIBED JUMP ON A SURFACE}

In this section we study some regularity results of harmonic maps in $\mathbb{R}^{3}$ with prescribed jump on a given surface. This will be crucial for the studying of the displacement in the presence of dislocations, since if a dislocation $C$ is the boundary of a surface $S$, then the displacement can be seen as a function with a fixed jump on the surface, equal to the Burgers vector associate to the dislocation.

In this work we will often need to make the following

Assumption 3.1. We consider a Lipschitz closed curve $C$, which is assumed simple, and thus non self-intersecting. Moreover we assume that $S$ is a bounded Lipschitz simple surface whose boundary is $C$, that is, $S$ is non self-intersecting and is homeomorphic to the disk $B_{1} \subset \mathbb{R}^{2}$.

Lemma 3.2. Let $C$ be a Lipschitz closed curve in $\mathbb{R}^{3}$ and $S$ a bounded Lipschitz surface with boundary $C$ and unit normal $N$ (satisfying Assumption 3.1). Let $b \in \mathbb{R}$. A solution of

$$
\begin{cases}\Delta u=0 & \text { in } \mathbb{R}^{3} \backslash S \\ {[u]:=u^{+}-u^{-}=b} & \text { on } S \\ {\left[\partial_{N} u\right]:=\partial_{N} u^{+}-\partial_{N} u^{-}=0} & \text { on } S\end{cases}
$$


is given by

$$
u(x)=-b \int_{S} \partial_{N} \Gamma\left(x^{\prime}-x\right) d \mathcal{H}^{2}\left(x^{\prime}\right),
$$

for $x \in \mathbb{R}^{3} \backslash S$, where $\Gamma$ is the solution in $\mathbb{R}^{3}$ of $\Delta \Gamma=\delta_{0}$. Moreover such solution is unique up to a harmonic map in $\mathbb{R}^{3}$.

By (3.2), it holds that

$$
\partial_{i} u(x)=-b \int_{S}\left(\frac{N_{i}}{\left\|x-x^{\prime}\right\|^{3}}-3 \frac{N \cdot\left(x-x^{\prime}\right)\left(x_{i}-x_{i}^{\prime}\right)}{\left\|x-x^{\prime}\right\|^{5}}\right) d \mathcal{H}^{2}\left(x^{\prime}\right) .
$$

Lemma 3.2 is a particular case of its general vector counterpart, whose proof can be found in [33]. Its statement is given in the following lemma, where $\mathbb{A}$ is a fourth-order elasticity tensor. So, the proof of Lemma 3.2 is straightforwardly obtained by letting $\mathbb{A}=\mathbb{I}_{2}$, the identity matrix.

Lemma 3.3. Let $\mathcal{L}$ be a Lipschitz closed curve in $\mathbb{R}^{3}$ and $S$ a bounded Lipschitz surface with boundary $\mathcal{L}$ and unit normal $N$. Let $B \in \mathbb{R}^{3}$. The solution of

$$
\begin{cases}\operatorname{div}(\mathbb{A} \nabla w)=0 & \text { in } \mathbb{R}^{3} \backslash S \\ \llbracket w \rrbracket:=w^{+}-w^{-}=B & \text { on } S \\ \llbracket(\mathbb{A} \nabla w) N \rrbracket:=((\mathbb{A} \nabla w) N)^{+}-((\mathbb{A} \nabla w) N)^{-}=0 & \text { on } S\end{cases}
$$

is given componentwise by

$$
w_{i}(x)=-B_{j} \int_{S}\left(\mathbb{A} \nabla \Gamma\left(x^{\prime}-x \cdot\right) N\left(x^{\prime}\right)\right)_{i j} d \mathcal{H}^{2}\left(x^{\prime}\right),
$$

for $x \in \mathbb{R}^{3} \backslash S$, where $\Gamma$ is the solution in $\mathbb{R}^{3}$ of $\operatorname{div}(\mathbb{A} \nabla \Gamma)=\delta_{0} \mathbb{I}$.

\subsection{Pointwise and distributional properties of gradients of harmonic maps.}

\subsubsection{Growth condition for the gradient.}

Lemma 3.4. Let $C$ and $S$ be as in Lemma 3.2 and $u$ be the explicit solution of (3.1) given by (3.2). If $C$ is smooth, there exists a constant $c>0$ depending on the curvature of $C$, such that it holds

$$
\left|\partial_{i} u(x)\right| \leq c|b| l\left(1+\frac{1}{d(x, C)}\right) .
$$

Proof. Step 1. Let us first prove that the value of the derivative $\partial_{i} u(x)$ does not depend on the surface $S$ appearing in (3.1). Let indeed $S^{\prime}$ be another smooth surface that does not contain the point $x$ and has $C$ as boundary. For simplicity let us suppose it is disjoint from $S$. Let $u^{\prime}$ be the solution of (3.1) with $S^{\prime}$ replacing $S$ and let $A$ be the open set enclosed by $S$ and $S^{\prime}$. By formula (3.2), $\left(u-u^{\prime}\right)(x)=c+b \int_{\partial A} \partial_{N}^{\prime} \Gamma\left(x^{\prime}-x\right) d \mathcal{H}^{2}\left(x^{\prime}\right)=c+b \chi_{A}(x)$, the second equality being a consequence of the Divergence theorem. In particular we see that $u-u^{\prime}$ is constant in a neighborhood of $x$, so that $\partial_{i} u(x)=\partial_{i} u^{\prime}(x)$. By approximation, we can also extend this to the case of Lipschitz surface $S^{\prime}$, and then to every rectifiable current $S^{\prime}$ with $\partial S^{\prime}=C$ whose support is outside a neighborhood of $x$.

Step 2. Let $d=d(x, C)$ be the distance form $x$ to $C$, let $\kappa$ be the maximum curvature of $C$, and let us denote by $R:=\kappa^{-1}$ the minimal radius of curvature. Let $B_{R}(x)$ be a ball with radius $R$ and center $x$, let $P$ be the point in $C$ such that $d=d(x, P)$, let $O$ be the point on the line $P x$, on the $x$ side, such that $d(O, P)=R$, and let $B_{R}(O)$ be a ball with radius $R$ and center $O$ (see figure $1)$. Let $\pi_{R}: \mathbb{R}^{3} \rightarrow \partial B_{R}(x)$ be the orthogonal projection onto the sphere $\partial B_{R}(x)$ and let $C_{R}$ be the image of $C$ throughout $\pi_{R}$. Let us consider the Lipschitz homotopy $\Phi:[0,1] \times[0, l] \rightarrow \Omega$ such that $\Phi(0,[0, l])=C, \Phi(1,[0, l])=C_{R}$, and $\Phi(\cdot, t)$ is affine for all $t \in[0, l]$. Then $E:=\Phi_{\sharp} \llbracket[0,1] \times[0, l] \rrbracket$ is a rectifiable current with boundary $C \cup C_{R}$. Let $D$ be an integral current in $B_{R}(x)$ with boundary $-C_{R}$. The rectifiable current $S^{\prime}:=E+D$ has boundary $C$, so we can consider the map $u^{\prime}$ solution of (3.1) with $S$ replaced by $S^{\prime}$. 


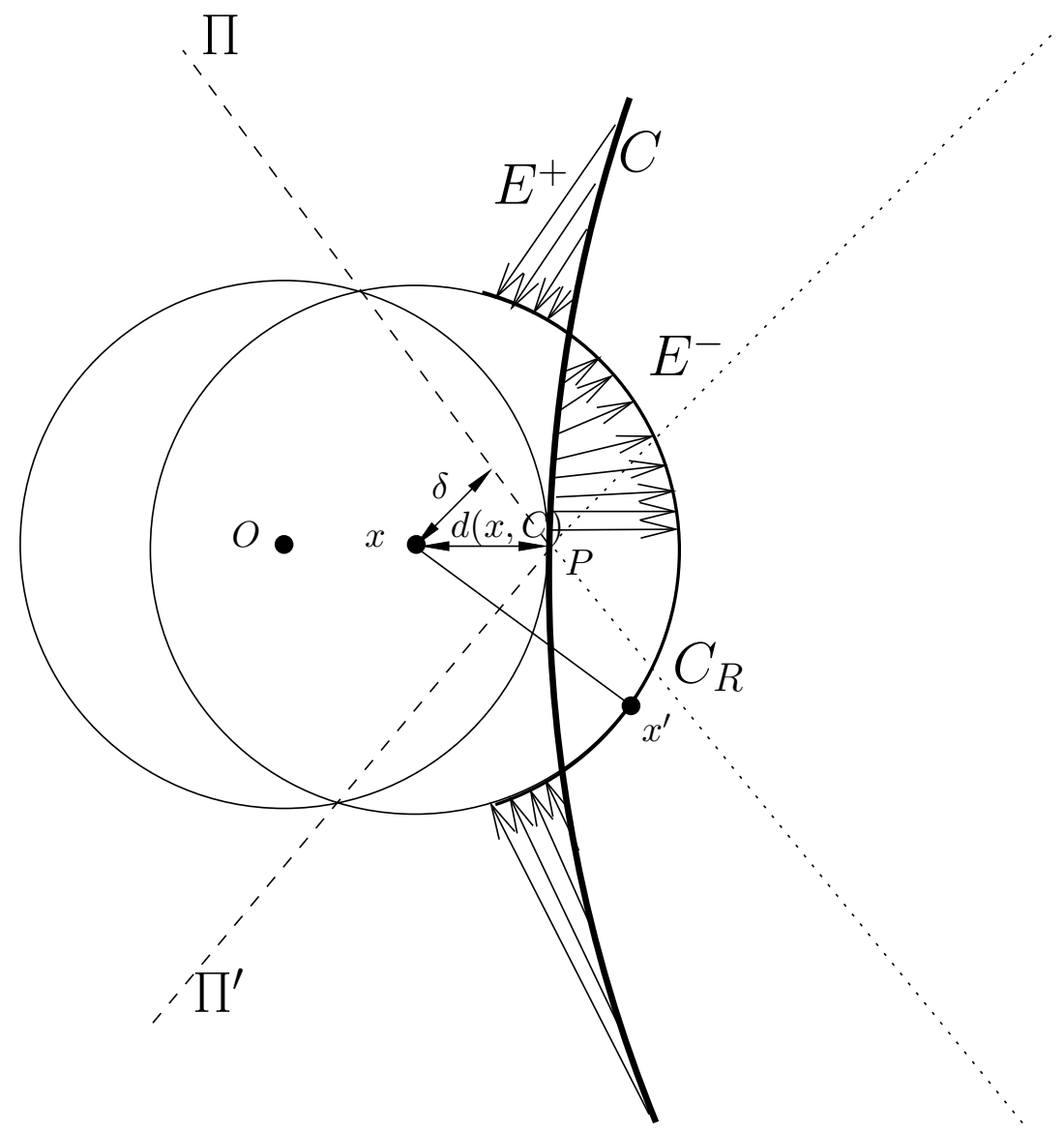

Figure 1. Geometry

Step 3. We claim that we can choose $D$ in such a way that its total mass $M(D)$ is bounded by $l R$. Indeed, since $C_{R}$ is an integral closed 1-current, it decomposes as $C_{R}=\sum_{i} C_{R}^{i}$, with $\sum_{i} l_{i}=$ $M\left(C_{R}\right) \leq l+2 \pi R$, where $l_{i}$ is the length of $C_{R}^{i}$, with $l$ the total length of $C$. Notice that the presence of the term $2 \pi R$ is justified as follows: $\pi_{R}$ is 1-Lipschitz outside $B_{R}$, whereas it is not Lipschitz inside. So we must estimate the inner part of $C$ separately. Since the maximum curvature of $C$ is assigned, it is seen that the part of $C$ inside $B_{R}$ is bounded and its projection on $B_{R}$ does not exceed $2 \pi R$. Having said that, by the isoperimetric inequality on $\partial B_{R}(x)$, it follows that

$$
l_{i}^{2} \geq 4 \pi A_{i}-\frac{A_{i}^{2}}{R^{2}}
$$

with $A_{i}$ the minimal area enclosed by $C_{R}^{i}$ (see Remark 3.5), so that $A_{i} \leq 2 \pi R^{2}$. It follows by simple computations that $A_{i} \leq 2 \pi R^{2}-R \sqrt{4 \pi^{2} R^{2}-l_{i}^{2}}$ and since $\sqrt{4 \pi^{2} R^{2}-l_{i}^{2}} \geq 2 \pi R-l_{i}$ if $l_{i} \leq 2 \pi R$, one deduces by mere substitution that $A_{i} \leq R l_{i} \wedge 2 \pi R^{2}$. As a consequence, $\sum_{i} A_{i} \leq l R$, and hence $M(D)<l R$. 
Step 4. Now, to compute $\left|\partial_{i} u(x)\right|$ we will use formula (3.3), integrating over $E+D$. Integration over $D$ can be estimated as follows:

$$
\begin{aligned}
\left|\partial_{i} u(x)\right| & =\left|b \int_{D}\left(\frac{N_{i}}{\left|x-x^{\prime}\right|^{3}}-3 \frac{N \cdot\left(x-x^{\prime}\right)\left(x_{i}-x_{i}^{\prime}\right)}{\left|x-x^{\prime}\right|^{5}}\right) d \mathcal{H}^{2}\left(x^{\prime}\right)\right| \\
& \leq \frac{4|b|}{R^{3}} \int_{D} d \mathcal{H}^{2}\left(x^{\prime}\right) \leq \frac{4|b| l}{R^{2}}
\end{aligned}
$$

since by Step $3, M(D) \leq l R$.

Let us now compute the integration over $E$. Observe that, by construction of $E$, it holds that its normal $N$ at a point $x^{\prime}$ satisfies $N \perp\left(x-x^{\prime}\right)$, and hence (3.3) yields

$$
\left|\partial_{i} u(x)\right| \leq|b|\left|\int_{E} \frac{N_{i}}{\left|x-x^{\prime}\right|^{3}} d \mathcal{H}^{2}\left(x^{\prime}\right)\right| .
$$

Let $E^{+}$(resp., $E^{-}$) be the part of $E$ outside $B_{R}(x)$ (inside, respectively). The integration can be performed by polar coordinates $(s, \rho)$ centered at $x$, where $s$ can be seen as an arc parameter on $C_{R}$. The integral of $(3.3)$ over $E^{+}$is bounded by

$$
\int_{C_{R}} d s \int_{R}^{+\infty} \frac{|b|}{\rho^{3}} d \rho \leq \frac{l|b|}{2 R^{2}} .
$$

More delicate is the computation of the integral over $E^{-}$(see Figure 1). Let $C^{-}$be the part of $C$ inside $B_{R}(x)$. Let us assume that $C^{-}$has $n$ connected components. Let $C_{1}^{-}$be one of this components. By the regularity hypotheses on $C$ and since $B_{R}(O)$ is tangent to $C$ at $P$, one has that $C$ stays outside of $B_{R}(O)$. Let $P_{1}$ and $P_{2}$ be the two points on $\partial B_{R}(0) \cap \partial B_{R}(x)$ belonging to the plane containing $0, x$, and the tangent vector $\tau$ to $C$ at $P$. Due to the boundedness of the curvature of $C$, it can be easily observed that the integral of (3.3) on the component $C_{1}^{-}$of $C^{-}$is maximal when $C_{1}^{-}$is planar and remains in the circumference obtained intersecting $B_{R}(0)$ with the plane containing $0, x$, and the tangent vector $\tau$ (the sheet plane in Figure 1). In such a case $C_{1}^{-}$is the arc of circumference passing for $P$ and with endpoints $P_{1}$ and $P_{2}$. Moreover, $E^{-}$is planar and is contained into two half-planes $\Pi$ and $\Pi^{\prime}$ passing through $P$, containing the tangent $\tau$, and with boundaries the lines $\overline{P_{1} P}$ and $\overline{P_{2} P}$, respectively (in Figure 1 ).

Now we estimate the integral on every connected components of $C^{-}$, and then multiply the obtained value by $n$ in order to get a right estimate. Since the half-planes $\Pi$ and $\Pi^{\prime}$ are constructed in such a way that $E^{-}$is contained in their union, the integral

$$
\int_{E^{-}} \frac{|b|}{d\left(x, x^{\prime}\right)^{3}} d \mathcal{H}^{2}\left(x^{\prime}\right)
$$

is bounded by the same integral over $\Pi \cup \Pi^{\prime}$. In particular, by symmetry, it holds

$$
\int_{E^{-}} \frac{|b|}{d\left(x, x^{\prime}\right)^{3}} d \mathcal{H}^{2}\left(x^{\prime}\right) \leq 2 \int_{\Pi} \frac{|b|}{d\left(x, x^{\prime}\right)^{3}} d \mathcal{H}^{2}\left(x^{\prime}\right) .
$$

If the distance $d(x, \Pi)=\delta$ the right-hand side is easily computed by integrating in planar polar coordinates with $\delta \leq r=d\left(x, x^{\prime}\right)<\infty$, and is equal to $\frac{2 \pi|b|}{\delta}$. Since by construction $\delta \geq 2^{-1 / 2} d(x, C)$, it holds

$$
\int_{E^{-}} \frac{|b|}{d\left(x, x^{\prime}\right)^{3}} d \mathcal{H}^{2}\left(x^{\prime}\right) \leq|b| \frac{2 \pi \sqrt{2}}{d(x, C)} .
$$

Now we have to multiply this by $n$, the number of connected components of $C^{-}$. We claim that $n \leq l /(\pi R)$. Indeed, consider a single connected component and one of its point $P$ inside $B_{R}(x)$. With elementary geometric arguments it is seen that, due to the boundedness of the curvature, as observed, it could stay inside $B_{R}(x) \backslash B_{R}(O)$ for a length at most $\pi R$. On the other hand, if it goes 
out of $B_{R}(x) \backslash B_{R}(O)$, then it cannot come back before spending a length of at least $\pi R$ outside. Then the claim follows, and it follows

$$
\int_{E^{-}} \frac{|b|}{d\left(x, x^{\prime}\right)^{3}} d \mathcal{H}^{2}\left(x^{\prime}\right) \leq \frac{2 \sqrt{2}}{R} \frac{|b| l}{d(x, C)}
$$

Summing all the bounds obtained so far we finally get

$$
\left|\partial_{i} u(x)\right| \leq 2 \sqrt{2} \frac{|b| l \kappa}{d(x, C)}+\frac{9}{2}|b| l \kappa^{2}
$$

from which the thesis follows.

Remark 3.5. In the previous proof we can choose the $A_{i}$ with minimal area as follows. Let $C_{R}^{i}$ be a simple loop in $B_{R}(x)$, let $P \notin C_{R}^{i}$ be a point on $\partial B_{R}(x)$. We construct an homotopy $\Psi^{P}$ : $[0,1] \times[0,2 \pi] \rightarrow \partial B_{R}(x)$ that satisfies $\Psi^{P}(0, \cdot) \equiv P$ and $\Psi^{P}(1,[0,2 \pi])=C_{R}^{i}$, and we can consider the current $\Psi_{\sharp}^{P} \llbracket[0,1] \times[0,2 \pi] \rrbracket$. Then we can set $A^{i}:=\Psi_{\sharp}^{P} \llbracket[0,1] \times[0,2 \pi] \rrbracket$, where $P$ is chosen in such a way that $\Psi_{\sharp}^{P} \llbracket[0,1] \times[0,2 \pi] \rrbracket$ has minimal mass.

Remark 3.6. In Lemma 3.4 we also proved that the integral in (3.2) does not depend on the particular surface $S$, but only on its boundary $C$.

Lemma 3.7. Let $b \in 2 \pi \mathbb{Z}$. Then the solution $u$ of (3.1) in (3.2) belongs to $\mathcal{C}^{\infty}(\Omega \backslash C, \mathbb{T})$ and it is harmonic in $\Omega \backslash C$.

Proof. As we have proved in the step 1 of Lemma 3.4, if we choose a surface $S^{\prime}$ with boundary $C$ disjoint from $S$, and denote by $u^{\prime}$ the corresponding solution of $(3.1)$, then $u-u^{\prime}=b \chi_{A}$, with $A$ the open set with boundary $S \cup S^{\prime}$. Since $b \in 2 \pi \mathbb{Z}$ we see that $u=u^{\prime}$ as a map into $\mathbb{T}$. Moreover if $x \notin S$ then $u$ is smooth at $x$, so in particular, up to change the surface $S$, we obtain that it belongs to $\mathcal{C}^{\infty}(\Omega \backslash C, \mathbb{T})$ and $u$ is harmonic at $x$ for all $x \notin C$.

Corollary 3.8. Let $C$ be the union of $N>0$ smooth closed curves $C_{k}$, let $S$ be the union of the corresponding surfaces $S_{k}$ with boundary $C_{k}$ respectively, and let $u$ be the solution to (3.1) given by (3.2). Then (3.6) holds true.

Proof. Actually the same proof as of Lemma 3.4 applies.

Corollary 3.9. Let $\mathcal{L}$ and $S$ be as in Lemma 3.3 and $w$ be the explicit solution of (3.4). If $\mathcal{L}$ is smooth, there exists a constant $c>0$ depending on the curvature of $C$, such that it holds

$$
\left|\partial_{j} w_{i}(x)\right| \leq \operatorname{cbl}\left(1+\frac{1}{d(x, C)}\right), \quad 1 \leq i, j \leq 3 .
$$

3.1.2. Finer description of the singularity.

Lemma 3.10. Let $S$ and $C$ be as above. Let $u$ be the solution to

$$
\left\{\begin{array}{cc}
\Delta u=0 & \text { in } \quad \mathbb{R}^{3} \backslash S \\
u^{+}-u^{-}=1 & \text { on } S \\
\partial_{N} u^{+}-\partial_{N} u^{-}=0 & \text { on } S,
\end{array}\right.
$$

given by (3.2) with $b=1$. Then, if $U$ is a tubular neighborhood of $C$, for all $(\rho, \theta, \tau) \in U$ with $\theta \neq 0$, it holds

(i) there exists the limit $\lim _{\epsilon \rightarrow 0^{+}} u(\epsilon \rho, \theta, \tau)=u\left(0^{+}, \theta, \tau\right)=\frac{\theta}{2 \pi}+c$, where $c$ is a constant independent of $\theta$.

(ii) $\lim _{\epsilon \rightarrow 0^{+}}\left|\partial_{\tau} u(\epsilon \rho, \theta, \tau)\right|<c<+\infty$ for some constant $c>0$ that depends only on the curve $C$.

(iii) $\lim _{\epsilon \rightarrow 0^{+}}\left|\partial_{\rho} u(\epsilon \rho, \theta, \tau)\right|<c<+\infty$ for some constant $c>0$ that depends only on the curve $C$. 
Proof. With no loss of generality we suppose that the curve $C$ which represents the boundary of $S$ passes through the origin of an Euclidean coordinate system where it is tangent to the $z$-axis. Moreover we choose the coordinates $x_{1}$ and $x_{2}$ in such a way that $x_{1}=\rho \cos \theta$ and $x_{2}=\rho \sin \theta$, so that it follows that the point $(\epsilon \rho, \theta, z)$ coincides with $\left(\epsilon x_{1}, \epsilon x_{2}, z\right)$. For simplicity we take $z=0$ and denote $x=\left(x_{1}, x_{2}, 0\right)$, while $S$ is orthogonal to the $x_{2}$-axis in 0 . From Lemma 3.2 we have the following explicit formula

$$
u(\epsilon \rho, \theta, 0)=u\left(\epsilon x_{1}, \epsilon x_{2}, 0\right)=-\int_{S} \partial_{N} \Gamma\left(x^{\prime}-\epsilon x, y^{\prime}-\epsilon y, z^{\prime}\right) d \mathcal{H}^{2}\left(x^{\prime}, y^{\prime}, z^{\prime}\right),
$$

with the change of variables $\left(\epsilon x_{1}^{\prime \prime}, \epsilon x_{2}^{\prime \prime}, \epsilon z^{\prime \prime}\right)=\left(x_{1}^{\prime}, x_{2}^{\prime}, z^{\prime}\right)$ we obtain

$$
u\left(\epsilon x_{1}, \epsilon x_{2}, 0\right)=-\int_{\frac{1}{\epsilon} S} \partial_{N} \Gamma\left(x_{1}^{\prime \prime}-x_{1}, x_{2}^{\prime \prime}-x_{2}, z^{\prime \prime}\right) d \mathcal{H}^{2}\left(x_{1}^{\prime \prime}, x_{2}^{\prime \prime}, z^{\prime \prime}\right),
$$

where we have used the explicit expression of $\Gamma$. Letting $\epsilon$ go to zero we obtain

$$
\lim _{\epsilon \rightarrow 0^{+}} u(\epsilon \rho, \theta, z)=-\int_{\Pi_{0}} \partial_{N} \Gamma\left(x^{\prime \prime}-x\right) d \mathcal{H}^{2}\left(x^{\prime \prime}\right),
$$

where $\Pi_{0}$ is the half-plane $\left\{z=x_{2}=0, x_{1}>0\right\}$ and we have used the shorter notation $x^{\prime \prime}=$ $\left(x_{1}^{\prime \prime}, x_{2}^{\prime \prime}, z^{\prime \prime}\right)$. Thanks to Lemma 3.2 , we see that the right-hand side coincides with $u\left(x_{1}, x_{2}, 0\right)$, where $u$ is the solution of (3.11) with $S=\Pi_{0}$. But it is well known that such solution is given by, in cylindrical coordinates, $u(\rho, \theta, z)=\frac{\theta}{2 \pi}+c$ for a fixed constant $c$. In particular we have $\lim _{\epsilon \rightarrow 0^{+}} u(\epsilon \rho, \theta, z)=\frac{\theta}{2 \pi}+c$.

To prove statement (ii) we use the explicit expression (3.3), which reads, after the change of variables $x^{\prime}=\epsilon x^{\prime \prime}$ (here, again with abuse of notations, $\partial_{z} u=\partial_{\tau} u$ at $x=0$ ),

$$
\partial_{z} u\left(\epsilon x_{1}, \epsilon x_{2}, 0\right)=-\frac{1}{\epsilon} \int_{\frac{1}{\epsilon} S}\left(\frac{N_{z}}{\left|x-x^{\prime \prime}\right|^{3}}-3 \frac{N \cdot\left(x-x^{\prime \prime}\right)\left(z-z^{\prime \prime}\right)}{\left|x-x^{\prime \prime}\right|^{5}}\right) d \mathcal{H}^{2}\left(x^{\prime \prime}\right) .
$$

We fix $R>0$ and consider the ball $B_{\epsilon}$ with center $\left(\epsilon x_{1}, \epsilon x_{2}, 0\right)$ and radius $R$. We then write the last integral as

$$
\begin{aligned}
& -\frac{1}{\epsilon} \int_{\frac{1}{\epsilon} S \cap B_{\epsilon}}\left(\frac{N_{z}}{\left|x-x^{\prime \prime}\right|^{3}}-3 \frac{N \cdot\left(x-x^{\prime \prime}\right)\left(z-z^{\prime \prime}\right)}{\left|x-x^{\prime \prime}\right|^{5}}\right) d \mathcal{H}^{2}\left(x^{\prime \prime}\right) \\
& -\frac{1}{\epsilon} \int_{\frac{1}{\epsilon} S \cap B_{\epsilon}^{c}}\left(\frac{N_{z}}{\left|x-x^{\prime \prime}\right|^{3}}-3 \frac{N \cdot\left(x-x^{\prime \prime}\right)\left(z-z^{\prime \prime}\right)}{\left|x-x^{\prime \prime}\right|^{5}}\right) d \mathcal{H}^{2}\left(x^{\prime \prime}\right),
\end{aligned}
$$

and thanks to Remark 3.6, up to choose $R$ small enough, we can assume that the normal $N$ to $S$ at $x^{\prime}$ is orthogonal to the vector $\left(\epsilon x-x^{\prime}\right)$ for all $x^{\prime} \in B_{\epsilon}$, that is, to $\left(x-x^{\prime \prime}\right)$ in $\frac{1}{\epsilon} B_{\epsilon}$, so that the integral above becomes

$$
\begin{aligned}
& -\frac{1}{\epsilon} \int_{\frac{1}{\epsilon} S \cap B_{\epsilon}} \frac{N_{z}}{\left|x-x^{\prime \prime}\right|^{3}} d \mathcal{H}^{2}\left(x^{\prime \prime}\right) \\
& -\frac{1}{\epsilon} \int_{\frac{1}{\epsilon} S \cap B_{\epsilon}^{c}}\left(\frac{N_{z}}{\left|x-x^{\prime \prime}\right|^{3}}-3 \frac{N \cdot\left(x-x^{\prime \prime}\right)\left(z-z^{\prime \prime}\right)}{\left|x-x^{\prime \prime}\right|^{5}}\right) d \mathcal{H}^{2}\left(x^{\prime \prime}\right) .
\end{aligned}
$$

Let us now estimate the second term in (3.13). In $B_{\epsilon}^{c}$ it holds $\left|\epsilon x-x^{\prime}\right|>R$, that is, $\left|x-x^{\prime \prime}\right|>\epsilon^{-1} R$, so it is easy to see that this term can be estimated by

$$
\frac{\mathcal{H}^{2}(S)}{R^{3}} \leq(\gamma+1) \frac{l^{2}}{R^{3}}
$$

where $l$ is the length of $C$ and $\gamma>0$ is the constant of the isoperimetric inequality, since $S$ can be chosen arbitrarily.

It remains to estimate the first term. Let us consider the plane $\Pi$ passing through 0 and tangent to the vector $\bar{z}$ and to $\overline{x-0}$, the unit vector tangent to the $z$-axis and the vector passing through $x$ and 0 , respectively. Let $\Pi^{+}$be the half-plane in $\Pi$ bounded by the axis $\hat{z}$ and not containing the point $x$. Thanks to the smoothness of $C$, we can assume that there exists a smooth one-to-one 
map $\Phi: \Pi^{+} \cap B_{\epsilon} \rightarrow S \cap B_{\epsilon}$, so that also $N \circ \Phi: \Pi^{+} \ni \hat{x}^{\prime} \mapsto N\left(x^{\prime}\right)$ is smooth, and then in $B_{\epsilon} \cap \Pi^{+}$we can use the Taylor expansion of $N \circ \Phi$ at 0 . Going back to the variable $x^{\prime}=\epsilon x^{\prime \prime}$ (and $\hat{x}^{\prime}:=\epsilon \hat{x}^{\prime \prime}$ ), we find that the first term in (3.13) reads

$$
-\int_{S \cap B_{\epsilon}} \frac{N\left(x^{\prime}\right)}{\left|\epsilon x-x^{\prime}\right|^{3}} d \mathcal{H}^{2}\left(x^{\prime \prime}\right)=-\int_{S \cap B_{\epsilon}} \frac{\nabla^{2} N(0) \hat{x}^{\prime} \cdot \hat{x}^{\prime}}{\left|\epsilon x-x^{\prime}\right|^{3}}+\frac{r_{N}\left(\left|\hat{x}^{\prime}\right|^{2}\right)}{\left|\epsilon x-x^{\prime}\right|^{3}} d \mathcal{H}^{2}\left(x^{\prime}\right) .
$$

The Taylor expansion of $\Phi$ at 0 provides $x^{\prime}=\hat{x}^{\prime}+\nabla^{2} \Phi(0) \hat{x}^{\prime} \cdot \hat{x}^{\prime}+r_{\Phi}\left(\hat{x}^{\prime}\right)$ and if $R$ is small enough we can assume that $\left|\nabla^{2} \Phi(0) \hat{x}^{\prime} \cdot \hat{x}^{\prime}+r_{\Phi}\left(\hat{x}^{\prime}\right)\right|<\frac{1}{2}\left|\hat{x}^{\prime}\right|$. Note that, since $C$ is smooth, we can find such a $R>0$ satisfying the last inequality globally, i.e., $R$ is independent of the point $x$. In particular we find $\left|\epsilon x-x^{\prime}\right|>\left|\epsilon x-\hat{x}^{\prime}\right|-\left|\nabla^{2} \Phi(0) \hat{x}^{\prime} \cdot \hat{x}^{\prime}+r_{\Phi}\left(\hat{x}^{\prime}\right)\right|>\left|\hat{x}^{\prime}\right|-\frac{1}{2}\left|\hat{x}^{\prime}\right|=\frac{1}{2}\left|\hat{x}^{\prime}\right|$ for all $\epsilon>0$, so that the integral is bounded by

$$
\int_{\Pi^{+} \cap B_{\epsilon}} \frac{\left|\nabla^{2} N(0) \hat{x}^{\prime} \cdot \hat{x}^{\prime}\right|}{\left|\hat{x}^{\prime}\right|^{3}}+\frac{r_{N}\left(\left|\hat{x}^{\prime}\right|^{2}\right)}{\left|\hat{x}^{\prime}\right|^{3}} d \mathcal{H}^{2}\left(x^{\prime}\right)
$$

and taking into account that $R>0$ can be small as we want, we can also assume that $\left|r_{N}\left(\hat{x}^{\prime}\right)\right|<$ $\left|\nabla^{2} N(0) \hat{x}^{\prime} \cdot \hat{x}^{\prime}\right|$, whereby the last integral can be estimated by

$$
C_{0} \int_{\Pi^{+} \cap B_{\epsilon}} \frac{1}{\left|\hat{x}^{\prime}\right|} d \mathcal{H}^{2}\left(\hat{x}^{\prime}\right),
$$

where the constant $C_{0}$ is independent of $R$ and $x$. Taking the limit as $\epsilon \rightarrow 0$ we infer, by the monotone convergence theorem, that

$$
C_{0} \int_{\Pi^{+} \cap B(0, R)} \frac{1}{\left|\hat{x}^{\prime}\right|} d \mathcal{H}^{2}\left(\hat{x}^{\prime}\right),
$$

which is uniformly bounded. Now, since the value of $R$ is independent of the point $x$ but only depends on the geometry of the curve $C$, we achieved the proof.

Statement (iii) can be proved taking into account that choosing $R$ small enough a formula similar to (3.13) holds, and then arguing as for statement (ii).

Remark 3.11. Let us point out that Lemma 3.10 still holds true if we do not assume that $C$ is connected. Indeed if $C$ is the union of a finite family of smooth closed curves, the surface $S$ will be the union of a finite family of smooth surfaces and the arguments used in the proof of Lemma 3.10 still work.

Remark 3.12. The curve regularity required in Lemma 3.10 is $W^{3, \infty}$, because of estimates such as (3.14). Note also that $C_{0}$ depends on the curve curvature.

3.2. Distributional properties. In this section and in the following we often deal with BV functions $u$. In this case, if not otherwise advised, we will always denote by $D u$ the distributional derivative of $u$, and by $\nabla u$ the absolutely continuous part of $D u$ with respect to the Lebesgue measure.

Lemma 3.13. Let $C$ be a closed Lipschitz curve in $\Omega$ and let $b \in 2 \pi \mathbb{Z}^{3}$. Then for any Lipschitz surface $S$ with boundary $C$, every solution $u$ to (3.1) belongs to $B V^{p}\left(\Omega, \mathbb{R}^{3}\right)$ with $1 \leq p \leq \frac{3}{2}$, satisfies Div $\nabla u=0$ and - Curl $\nabla u=b \otimes \mathcal{L}$ as distributions, with $\nabla u$ the part of the gradient of $u$ that is absolutely continuous with respect to the Lebesgue measure. Moreover if the curve $C$ is smooth, then $\nabla u \in L^{p}\left(\Omega, \mathbb{R}^{3 \times 3}\right)$ for all $1 \leq p<2$.

Proof. Let $u$ be a solution to (3.1) and let us first assume $C$ smooth. By (3.6), $\nabla u \in L^{p}\left(\Omega, \mathbb{R}^{3 \times 3}\right)$ for $p<2$. It has been shown that $u$ is smooth outside $S$ where it has a jump of amplitude $b$. In particular $u$ belongs to $S B V\left(\Omega, \mathbb{R}^{3}\right)$ and its distributional derivative is given by

$$
\langle D u, \varphi\rangle:=-\langle u, \operatorname{div} \varphi\rangle=S(\varphi)+\langle\nabla u, \varphi\rangle,
$$

for all $\varphi \in \mathcal{D}\left(\Omega, \mathbb{R}^{3 \times 3}\right)$, where $S$ denotes the distribution $S(\varphi)=-\int_{S} N_{j} b_{i} \varphi_{i j} d \mathcal{H}^{2}$. 
Let us prove that $-\operatorname{Curl} \nabla u=\mathcal{L} \otimes b$. To this aim let us take $\psi \in \mathcal{D}\left(\Omega, \mathbb{R}^{3 \times 3}\right)$ and write

$$
\begin{aligned}
-\langle\operatorname{Curl} \nabla u, \psi\rangle & :=-\langle\nabla u, \operatorname{Curl} \psi\rangle=-\langle D u, \operatorname{Curl} \psi\rangle+S(\operatorname{Curl} \psi) \\
& =\int_{C} \tau_{j} b_{i} \psi_{i j} d \mathcal{H}^{1}=b \otimes \mathcal{L}(\psi),
\end{aligned}
$$

where the second equality follows from (3.15) with $\varphi=\operatorname{Curl} \psi$, and the third one by Stokes theorem.

We now prove that Div $\nabla u=0$. Again, we take $\psi \in \mathcal{D}\left(\Omega, \mathbb{R}^{3}\right)$ and write

$$
-\langle\operatorname{Div} \nabla u, \psi\rangle:=\langle\nabla u, \nabla \psi\rangle=\langle D u, \nabla \psi\rangle-S(\nabla \psi),
$$

and using the explicit formula (3.2) for $u$ we obtain

$$
\begin{aligned}
\langle D u, \nabla \psi\rangle & =b_{k}\left\langle D_{i} \int_{S} \partial_{N} \Gamma_{k}\left(x^{\prime}-\cdot\right) d \mathcal{H}^{2}\left(x^{\prime}\right), D_{i} \psi\right\rangle \\
& =-\int_{S} b_{k}\left\langle\Delta \Gamma_{k}\left(x^{\prime}-\cdot\right), D_{j} \psi N_{j}\right\rangle d \mathcal{H}^{2}\left(x^{\prime}\right) \\
& =-b_{k} \int_{S} \partial_{N} \psi_{k}\left(x^{\prime}\right) d \mathcal{H}^{2}\left(x^{\prime}\right)=S(\nabla \psi),
\end{aligned}
$$

so that plugging the last identity in (3.17) we obtain Div $\nabla u=0$.

Let us now treat the general case. We proceed by approximation, so let $C_{n}$ be a sequence of curves converging uniformly and in the sense of currents to $C$, let $S_{n}$ be surfaces converging in the same sense to $S$, and let $u_{n}$ be the corresponding solutions. Let $V_{n}$ be the solution to the system

$$
\left\{\begin{array}{cccc}
-\operatorname{Curl} U & = & -b \otimes \mathcal{L}_{n} & \text { in } \hat{\Omega} \\
\operatorname{div} U & = & 0 & \text { in } \hat{\Omega} \\
U N & = & 0 & \text { on } \partial \hat{\Omega},
\end{array},\right.
$$

which, by Theorem 2.6 also satisfies $\|U\|_{L^{p}} \leq C|\mu|(\hat{\Omega})$, with $C=C(\hat{\Omega})$, so that we infer $\left\|V_{n}\right\|_{L^{p}(\Omega)} \leq$ $|b|\left|\mathcal{L}_{n}\right|$ for $1 \leq p \leq \frac{3}{2}$. So far we have proved that $\nabla u_{n}$ differs from $-V_{n}^{T}$ by the gradient of a harmonic map whose boundary datum at $\partial \Omega$ are bounded since $d\left(C_{n}, \partial \Omega\right)>\delta>0$ (this can be seen computing $\nabla u_{n} N$ by formula (3.3)). In particular we find that there are constants $C_{1}, C_{2}>0$ such that $\left\|\nabla u_{n}\right\|_{L^{p}(\Omega)} \leq C_{1}|b|\left|\mathcal{L}_{n}\right|+C_{2}$ for $1 \leq p \leq \frac{3}{2}$. Now it is easy to see that $u_{n} \rightarrow u$ weakly* in $B V^{p}\left(\Omega, \mathbb{R}^{3}\right)$, and the conclusion easily follows.

Remark 3.14. In order to prove that Div $\nabla u=0$, we might also argue as follows. Let $\hat{S} \supset S$ such that $\hat{S}$ separates $\Omega$ in two parts $\Omega^{-}$and $\Omega^{+}$. Then for every test function $\varphi \in \mathcal{C}_{c}^{\infty}\left(\Omega, \mathbb{R}^{3}\right)$ it holds

$$
\begin{aligned}
& \int_{\Omega} \nabla u \nabla \varphi d x=\int_{\Omega^{+}} \nabla u \nabla \varphi d x+\int_{\Omega^{-}} \nabla u \nabla \varphi d x= \\
& -\int_{\Omega^{+}} \operatorname{Div} \nabla u \varphi d x-\int_{\Omega^{-}} \operatorname{Div} \nabla u \varphi d x+\int_{\hat{S}^{+}} \partial_{N} u^{+} \varphi d x-\int_{\hat{S}^{-}} \partial_{N} u^{-} \varphi d x=0 .
\end{aligned}
$$

Remark 3.15. The statement of Lemma 3.13 readily applies to the case of $C$ being a finite union of Lipschitz curves.

According to Corollary 3.3, the counterpart of Lemma 3.13 for a vector-valued displacement in linear elasticity reads as follows:

Corollary 3.16. Let $C \subset \Omega$ be the union of a finite number of smooth dislocation loops and $S \subset \Omega$ a smooth surface enclosed by C. Referring to Lemma 3.3, let $w$ be the solution of

$$
-\operatorname{div}(\mathbb{A} \nabla w)=0 \quad \text { in } \quad \mathbb{R}^{3} \backslash S, \quad \llbracket w \rrbracket=b \in 2 \pi \mathbb{Z}^{3}, \quad \llbracket(\mathbb{A} \nabla w) N \rrbracket=0 \quad \text { on } \quad S,
$$


given by (3.5). Then $w \in S B V\left(\Omega, \mathbb{R}^{3}\right), \nabla w \in L^{p}\left(\Omega, \mathbb{R}^{3}\right)$ for $1 \leq p<2$ and $-\operatorname{Curl} \nabla w=\Lambda_{\mathcal{L}}^{T}$ in the distribution sense, where $\nabla w$ is the absolutely continuous part of the distributional derivative $D w$ in $\Omega$. Moreover $-\operatorname{div}(\mathbb{A} \nabla w)=0$ in $\mathbb{R}^{3} \backslash C, w \in \mathcal{C}^{\infty}\left(\Omega \backslash \mathcal{L}, \mathbb{R}^{3}\right)$ and it holds

$$
|\nabla w(x)| \leq c|b| l\left(1+\frac{1}{d(x, C)}\right),
$$

with $c$ a constant depending on the maximal curvature of $C$, and $l$ its length.

The bound (3.19) is proved as in Lemma 3.4, since $\mathbb{A}$ is a bounded tensor.

\section{Main Result: GRAPH Boundary of $\mathbb{T}^{3}$-VALUED haRMONIC MAPS}

Preliminary notions. We introduce the following notation. For all $b \in \mathbb{R}^{3}$ we define the 1-current $\vec{b} \in \mathcal{D}_{1}\left(\mathbb{T}^{3}\right)$ as

$$
\vec{b}(\omega):=-\frac{1}{2 \pi} \int_{0}^{2 \pi}\left\langle\omega\left(\frac{b_{1} \theta}{2 \pi}, \frac{b_{2} \theta}{2 \pi}, \frac{b_{3} \theta}{2 \pi}\right), b\right\rangle d \theta,
$$

for any 1-form $\omega \in \mathcal{D}^{1}\left(\mathbb{T}^{3}\right)$. It is easy to see that $M(\vec{b})=|b|$. The fact that we are on the torus, i.e., $\omega$ is $2 \pi$-periodic on $\mathbb{R}^{3}$, implies that $\vec{b}$ is a closed current whenever $b \in 2 \pi \mathbb{Z}^{3}$. Moreover it is convenient to define, for all $b \in \mathbb{R}^{3}$ and all $r \in \mathbb{R}^{3}$, the 1-current $\vec{b}_{r} \in \mathcal{D}_{1}\left(\mathbb{T}^{3}\right)$ as

$$
\vec{b}_{r}(\omega):=-\frac{1}{2 \pi} \int_{0}^{2 \pi}\left\langle\omega\left(r_{1}+\frac{b_{1} \theta}{2 \pi}, r_{2}+\frac{b_{2} \theta}{2 \pi}, r_{3}+\frac{b_{3} \theta}{2 \pi}\right), b\right\rangle d \theta,
$$

for any 1-form $\omega \in \mathcal{D}^{1}\left(\mathbb{T}^{3}\right)$. Also in this case $M\left(\vec{b}_{r}\right)=|b|$ for all $r \in \mathbb{R}^{3}$. Note that, if $b \in 2 \pi \mathbb{Z}^{3}$ and there is a real number $\delta$ such that $b=\delta r$, then the currents $\vec{b}=\vec{b}_{r}$ thanks to the periodicity of the forms in $\mathcal{D}^{1}\left(\mathbb{T}^{3}\right)$.

We denote by $\mathcal{L} \wedge \vec{b}$ the 2-current in $\Omega \times \mathbb{T}^{3}$ defined as

$$
\mathcal{L} \wedge \vec{b}(\omega)=-\frac{1}{2 \pi} \int_{C} \int_{0}^{2 \pi}\left\langle\omega\left(x, \frac{b \theta}{2 \pi}\right), \bar{\tau} \wedge \bar{b}\right\rangle d \theta d \mathcal{H}^{1}(x),
$$

for any 2-form $\omega \in \mathcal{D}^{2}\left(\Omega \times \mathbb{T}^{3}\right)$ (we mean $\bar{\tau}=(\vec{\tau}, 0) \in \mathbb{R}^{3} \times \mathbb{R}^{3}$, the tangent vector to $C$ in $\Omega \times \mathbb{R}^{3}$, and $\bar{b}=(0, b) \in \mathbb{R}^{3} \times \mathbb{R}^{3}$, with $\vec{\tau}$ the tangent vector to $C$ in $\Omega$ ).

The cylindrical neighborhood. Let $C$ be a closed loop of class $\mathcal{C}^{2}$. There is a cylindrical neighborhood $D_{R}$ of $C$, with cylindrical coordinates $(\rho, \theta, z) \in[0, R] \times[0,2 \pi] \times[0, l] / \sim$, where $\sim$ means that the coordinate $\theta=0$ (and $z=0$ ) is identified with $\theta=2 \pi$ (resp. $z=l$ ). The neighborhood $D_{R}$ is also parametrized by the coordinates $(x, y, z)$ by setting $x=\rho \cos \theta$ and $y=\rho \sin \theta$. Let $S$ be a smooth surface with boundary $C$ and such that $S \cap D_{R}$ coincides with the set $\{\theta=0\}$. We suppose Assumption 3.1 on the regularity of $S$. The tangent space to $\partial D_{R}$ is a 2-dimensional plane with orthonormal basis $\{\sigma, \tau\}$, with $\tau$ representing the vector tangent to the curve, and then corresponding to the $z$ coordinate, and $\sigma$ tangent to the section (orthogonal to $C$ ) of $\partial D_{R}$, namely corresponding to the coordinate $\theta$.

4.1. Statement of the main theorem. We are ready to prove the following:

Theorem 4.1. Let $S$ be a smooth surface in $\Omega$ whose boundary $C$ is a smooth and closed curve in $\Omega$, both satisfying Assumption 3.1. Let $b=\left(b_{1}, b_{2}, b_{3}\right) \in 2 \pi \mathbb{Z}^{3}$ and let $u=\left(u_{1}, u_{2}, u_{3}\right): \Omega \rightarrow \mathbb{R}^{3}$ be the map with $u_{i}$ given by $(3.2)$ with $b=b_{i}$. Then $\mathcal{G}_{u}$ is an integral current in $\mathcal{D}_{3}\left(\Omega \times \mathbb{T}^{3}\right)$ and its boundary is given by

for all $\omega \in \mathcal{D}^{2}\left(\Omega \times \mathbb{T}^{3}\right)$.

$$
\partial \mathcal{G}_{u}(\omega)=\mathcal{L} \wedge \vec{b}(\omega),
$$


Remark 4.2. For all $\omega \in \mathcal{D}^{2}\left(\Omega \times \mathbb{T}^{3}\right)$ let us write $\omega=\sum_{i=0}^{2} \omega^{i}$ where we have set

$$
\omega^{i}:=\sum_{\alpha:|\alpha|=i} \omega_{\alpha \beta} d x_{\alpha} \wedge d y_{\beta}
$$

In other words $\omega^{i}$ represents the components of $\omega$ whose coefficient $d x_{\alpha} \wedge d y_{\beta}$ has a $i$-dimensional horizontal component $\left(d x_{\alpha}\right)$ and a $2-i$ dimensional vertical component $\left(d y_{\beta}\right)$. Let $\Phi_{\theta}(x):=\left(x, \frac{b_{1} \theta}{2 \pi}, \frac{b_{2} \theta}{2 \pi}, \frac{b_{3} \theta}{2 \pi}\right)$ and define the tensor test function $\varphi^{\omega}$ associated to any form $\omega \in \mathcal{D}^{2}\left(\Omega \times \mathbb{T}^{3}\right)$ componentwise as

$$
\varphi_{\alpha \beta}^{\omega}:=\int_{0}^{2 \pi}\left\langle\omega^{1} \circ \Phi_{\theta}, e_{\alpha} \wedge \varepsilon_{\beta}\right\rangle d \theta,
$$

where $\alpha, \beta=1,2,3$. Then, our main result can be restated as

$$
\partial \mathcal{G}_{u}(\omega)=\Lambda_{\mathcal{L}}\left(\varphi^{\omega}\right):=\mathcal{L} \otimes b\left(\varphi^{\omega}\right),
$$

for all $\omega \in \mathcal{D}^{3}\left(\Omega \times \mathbb{T}^{3}\right)$, where $\varphi^{\omega}$ is defined by (4.6).

Corollary 4.3. Let $S$ be a Lipschitz surface in $\Omega$ whose boundary $C$ is a Lipschitz and closed curve in $\Omega$. Let $b=\left(b_{1}, b_{2}, b_{3}\right) \in 2 \pi \mathbb{Z}^{3}$ and let $u=\left(u_{1}, u_{2}, u_{3}\right): \Omega \rightarrow \mathbb{R}^{3}$ be a map with $u_{i}$ satisfying (3.2) with $b=b_{i}$. Then $\mathcal{G}_{u}$ is an integral current in $\mathcal{D}_{3}\left(\Omega \times \mathbb{T}^{3}\right)$ and (4.4) holds.

Proof. We proceed by approximation. Let $\left\{C_{k}\right\}_{k>0}$ be a sequence of smooth closed curves approximating $C$ (uniformly and in the sense of 1-currents) and let $\left\{S_{k}\right\}_{k>0}$ be smooth surfaces with boundary $\left\{C_{k}\right\}_{k>0}$ and converging (uniformly and in the sense of currents) to $S$. Let $u_{k}$ be maps as in Theorem 4.1 with $C$ replaced by $C_{k}$ and $S$ replaced by $S_{k}$. Thanks to the uniform convergence of $S_{k}$ to $S$ and using formula (3.2) we see that $u_{k}$ converges pointwise to $u$, and then strongly in $L^{p}\left(\Omega, \mathbb{T}^{3}\right)$. Since $C_{k}$ are converging uniformly to $C$ whose length is finite, the lengths of $C_{k}$ are uniformly bounded so the same argument employed in Lemma 3.13 gives a uniform bound in $L^{p}(\Omega)$, with $p<\frac{3}{2}$, for the $1 \times 1$ minors of $D u_{k}$, while the higher-order minors are all null. Therefore there are maps $v_{\bar{\alpha}}^{\beta^{\beta}} \in L^{p}(\Omega)$ such that, up to a subsequence, $M_{\bar{\alpha}}^{\beta}\left(D u_{k}\right) \rightarrow v_{\bar{\alpha}}^{\beta}$ weakly in $L^{p}(\Omega)$. Finally, the lengths of $C_{k}$ being uniformly bounded, Theorem 4.1 provides an uniform bound on the masses of $\partial \mathcal{G}_{u_{k}}$. Now Theorem 2.3 applies and implies that $u \in \mathcal{A}_{p}\left(\Omega, \mathbb{T}^{3}\right)$. In particular we have that $u_{k} \rightarrow u$ weakly in $\mathcal{A}_{p}\left(\Omega, \mathbb{T}^{3}\right)$, thus Lemma 2.2 implies that $\partial \mathcal{G}_{u_{k}} \rightarrow \partial \mathcal{G}_{u}$ as currents, and the fact that for $u_{k}$ the explicit form (4.4) holds true implies that it holds also at the limit, concluding the proof.

4.1.1. Proof of the main theorem: Theorem 4.1. In the sequel we will use the notation

$$
\Phi:=I d \times u: \Omega \rightarrow \Omega \times \mathbb{T}^{3} .
$$

Before proving Theorem 4.1 we state the following preliminary fact:

Lemma 4.4. Let $u$ be as in Theorem 4.1. Then $u \in \mathcal{A}_{p}\left(\Omega, \mathbb{T}^{3}\right)$ for all $1 \leq p<2$.

Proof. Lemma 3.7 shows that $u$ is well-defined in $\mathbb{T}^{3}$. In order to prove that it belongs to $\mathcal{A}_{p}\left(\Omega, \mathbb{T}^{3}\right)$ we need to show that all its minors $M_{\bar{\alpha}}^{\beta}(D u)$ belong to $L^{p}(\Omega)$. Thanks to Lemma 3.4 it is easy to see that every $1 \times 1$-minor belongs to $L^{p}(\Omega)$. Moreover from Lemma 3.2 we have that $u_{1}, u_{2}$, and $u_{3}$ differ by a multiplicative constant, so that the rows of the matrix $D u$ are linearly dependent. In particular all the minors greater than $1 \times 1$ vanish, and the thesis follows.

Proof of Theorem 4.1. Let $u_{\epsilon}$ be the restriction of the map $u$ to $\Omega_{\epsilon}:=\Omega \backslash \bar{D}_{\epsilon}, u_{\epsilon}:=u\left\llcorner\Omega_{\epsilon}\right.$, where $D_{\epsilon}:=\{(\rho, \theta, z) \in[0, R] \times[0,2 \pi] \times[0, l] / \sim: \rho<\epsilon\}$. The graph $\mathcal{G}_{u_{\epsilon}}$ is the restriction of the graph $\mathcal{G}_{u}$ to the open set $\Omega_{\epsilon} \times \mathbb{T}^{3}$. Formula (2.6) and the Dominated Convergence Theorem readily implies that $\mathcal{G}_{u_{\epsilon}} \rightarrow \mathcal{G}_{u}$ as currents. As a consequence we find

$$
\partial \mathcal{G}_{u_{\epsilon}} \rightarrow \partial \mathcal{G}_{u}
$$


In order to compute explicitly the boundary of $\mathcal{G}_{u}$ we write $\partial \mathcal{G}_{u_{\epsilon}}(\omega)=\mathcal{G}_{u_{\epsilon}}(d \omega)$, for $\omega \in \mathcal{D}^{2}\left(\Omega \times \mathbb{R}^{3}\right)$. Lemma 3.7 implies that $u$ is smooth outside a neighborhood of $C$, so that we can apply the Stokes Theorem and find

$$
\partial \mathcal{G}_{u_{\epsilon}}(\omega)=\int_{\partial D_{\epsilon}}\left\langle\omega \circ \Phi, \frac{\partial \Phi}{\partial \sigma} \wedge \frac{\partial \Phi}{\partial \tau}\right\rangle d \mathcal{H}^{2}(x)
$$

where $(\sigma, \tau)$ is an orthogonal coordinate system in the tangent space to $\partial D_{\epsilon}$. The gradient of $\Phi$ reads

$$
(D \Phi)=\left(\begin{array}{ccc}
1 & 0 & 0 \\
0 & 1 & 0 \\
0 & 0 & 1 \\
\frac{\partial u_{1}}{\partial x_{1}} & \frac{\partial u_{1}}{\partial x_{2}} & \frac{\partial u_{1}}{\partial x_{3}} \\
\frac{\partial u_{2}}{\partial x_{1}} & \frac{\partial u_{2}}{\partial x_{2}} & \frac{\partial u_{2}}{\partial x_{3}} \\
\frac{\partial u_{3}}{\partial x_{1}} & \frac{\partial u_{1}}{\partial x_{2}} & \frac{\partial u_{3}}{\partial x_{3}}
\end{array}\right) .
$$

holds

Let $\partial D_{\epsilon} \cong[0,2 \pi] \times[0, l] / \sim$ for all $(\theta, \tau) \in \partial D_{\epsilon}$. In the coordinate system $\left(\rho, \sigma, \tau, y_{1}, y_{2}, y_{3}\right)$ it

$$
\left(D\left(\Phi\left\llcorner\partial D_{\epsilon}\right)\right)=\left(\frac{\partial \Phi}{\partial \sigma}, \frac{\partial \Phi}{\partial \tau}\right)=\left(\begin{array}{cc}
0 & 0 \\
1 & 0 \\
0 & 1 \\
\frac{\partial u_{1}}{\partial \sigma} & \frac{\partial u_{1}}{\partial \tau} \\
\frac{\partial u_{2}}{\partial \sigma} & \frac{\partial u_{2}}{\partial \tau^{\tau}} \\
\frac{\partial u_{3}}{\partial \sigma} & \frac{\partial u_{3}}{\partial \tau}
\end{array}\right) .\right.
$$

If $\omega=\omega_{i j} d z_{i} \wedge d z_{j}$, with $1 \leq i<j \leq 6$, where we have defined $z_{1}=\rho, z_{2}=\sigma, z_{3}=\tau$, and $z_{k+3}=y_{k}$ for $k=1,2,3$, we can write

$$
\int_{\partial D_{\epsilon}}\left\langle\omega \circ \Phi, \frac{\partial \Phi}{\partial \sigma} \wedge \frac{\partial \Phi}{\partial \tau}\right\rangle d \mathcal{H}^{2}(x)=\int_{\partial D_{\epsilon}} \sigma(i, \bar{i}) \omega_{i j}(x, u(x)) \tilde{M}_{i}^{j}\left(D\left(\Phi\left\llcorner\partial D_{\epsilon}(x)\right)\right) d \mathcal{H}^{2}(x),\right.
$$

with $\tilde{M}_{i}^{j}\left(D\left(\Phi\left\llcorner\partial D_{\epsilon}(x)\right)\right)\right.$ being the minor of $D\left(\Phi\left\llcorner\partial D_{\epsilon}\right)\right.$ given by the $i$-th and $j$-th rows. From (4.9) we see that the $(2 \times 2)$-minors of $D\left(\Phi\left\llcorner\partial D_{\epsilon}\right)\right.$ which are nonzero are the only ones involving either the second or third row. So (4.10) reads

$$
\begin{aligned}
& \int_{\partial D_{\epsilon}}\left(\omega_{23}(x, u(x))-\sum_{k=4}^{6}\left(\omega_{2 k}(x, u(x)) \frac{\partial u_{k-3}}{\partial \tau}(x)+\omega_{3 k}(x, u(x)) \frac{\partial u_{k-3}}{\partial \sigma}(x)\right)\right) d \mathcal{H}^{2}(x)= \\
& \int_{\partial D_{\epsilon}} \omega_{23}(x, u(x)) d \mathcal{H}^{2}(x)-\sum_{k=4}^{6} \int_{0}^{2 \pi} \int_{0}^{l} \epsilon \tilde{\omega}_{2 k}(\epsilon, \theta, \tau, u(\epsilon, \theta, \tau)) \frac{\partial u_{k-3}}{\partial \tau}(\epsilon, \theta, \tau) d \tau d \theta \\
& \left.-\sum_{k=4}^{6} \int_{0}^{l} \int_{0}^{2 \pi} \epsilon \tilde{\omega}_{3 k}(\epsilon, \theta, \tau, u(\epsilon, \theta, \tau)) \frac{\partial u_{k-3}}{\partial \sigma}(\epsilon, \theta, \tau)\right) d \theta d \tau,
\end{aligned}
$$

where $\tilde{\omega}:=\omega \operatorname{det} \Psi$, with $\Psi:[0, \epsilon] \times[0,2 \pi] \times[0, l] \rightarrow D_{\epsilon}$ is the map of change of variables. Note that by the assumption of smoothness of $C$, we have that $\Psi$ is smooth and $\operatorname{det} \Psi=1$ on $C$. Now the first term of the right-hand side of (4.11) vanishes as $\epsilon \rightarrow 0$ since $\omega$ is bounded and $\mathcal{H}^{2}\left(\partial D_{\epsilon}\right) \rightarrow 0$. 
Integrating by parts the second term and using Lemma 3.10 we obtain

$$
\begin{aligned}
& \sum_{k=4}^{6} \int_{0}^{2 \pi} \epsilon \int_{0}^{l} \frac{\partial \tilde{\omega}_{2 k}}{\partial \tau}(\epsilon, \theta, \tau, u(\epsilon, \theta, \tau)) u_{k-3}(\epsilon, \theta, \tau) d \tau d \theta= \\
& =\sum_{k=4}^{6} \int_{0}^{2 \pi} \epsilon \int_{0}^{l} \frac{\partial \tilde{\omega}_{2 k}}{\partial \tau}(\epsilon, \theta, \tau, u(\epsilon, \theta, \tau))\left(\frac{\theta b_{k-3}}{2 \pi}+O(1)\right) d \tau d \theta \\
& +\sum_{k=4}^{6} \int_{0}^{2 \pi} \epsilon \int_{0}^{l} \sum_{h=1}^{3} \frac{\partial \tilde{\omega}_{2 k}}{\partial x_{3+h}}(\epsilon, \theta, \tau, u(\epsilon, \theta, \tau)) \frac{\partial u_{h}}{\partial \tau}(\epsilon, \theta, \tau)\left(\frac{\theta b_{k-3}}{2 \pi}+O(1)\right) d \tau d \theta
\end{aligned}
$$

where $|O(1)| \leq C$ as $\epsilon \rightarrow 0$, so that its absolute value can be estimated by $\epsilon\left\|\frac{\partial \tilde{\omega}_{2 k}}{\partial x_{i}}\right\|_{\infty} C$ thanks to Lemma 3.10, whereby this term vanishes as well as $\epsilon \rightarrow 0$.

As for the third term of (4.11), we first set

$$
\left.R(\epsilon):=-\sum_{k=4}^{6} \int_{0}^{2 \pi} \int_{0}^{l} \epsilon \Delta \tilde{\omega}_{3 k}(\epsilon, \theta, \tau, u(\epsilon, \theta, \tau)) \frac{\partial u_{k-3}}{\partial \sigma}(\epsilon, \theta, \tau)\right) d \tau d \theta
$$

with, recalling that $u_{k-3}\left(0^{+}, \theta, \tau\right)=\lim _{\epsilon \rightarrow 0^{+}} u(\epsilon \rho, \theta, \tau)=\frac{\theta}{2 \pi} b_{k}+c$,

$$
\Delta \tilde{\omega}_{3 k}(\epsilon, \theta, \tau, u(\epsilon, \theta, \tau)):=\tilde{\omega}_{3 k}(\epsilon, \theta, \tau, u(\epsilon, \theta, \tau))-\tilde{\omega}_{3 k}\left(\hat{\tau}, u_{k-3}\left(0^{+}, \theta, \tau\right)\right),
$$

where $\hat{\tau}:=(0,0, \tau)$. Since $\frac{\partial}{\partial \sigma}=\frac{1}{\epsilon} \frac{\partial}{\partial \theta}$, we obtain

$$
\begin{aligned}
& \left.-\sum_{k=4}^{6} \int_{0}^{l} \int_{0}^{2 \pi} \tilde{\omega}_{3 k}\left(\hat{\tau}, u_{k-3}\left(0^{+}, \theta, \tau\right)\right) \frac{\partial u_{k-3}}{\partial \theta}(\epsilon, \theta, \tau)\right) d \theta d \tau+R(\epsilon)= \\
& =-\left.\sum_{k=4}^{6} \int_{0}^{l} \tilde{\omega}_{3 k}\left(\hat{\tau}, \frac{b_{1} \theta}{2 \pi}+c, \frac{b_{2} \theta}{2 \pi}+c, \frac{b_{3} \theta}{2 \pi}+c\right) u_{k-3}(\epsilon, \theta, \tau)\right|_{0} ^{\theta=2 \pi} d \tau \\
& \left.+\sum_{k=4}^{6} \int_{0}^{l} \int_{0}^{2 \pi} \frac{d}{d \theta} \tilde{\omega}_{3 k}\left(\hat{\tau}, \frac{b_{1} \theta}{2 \pi}+c, \frac{b_{2} \theta}{2 \pi}+c, \frac{b_{3} \theta}{2 \pi}+c\right) u_{k-3}(\epsilon, \theta, \tau)\right) d \theta d \tau+R(\epsilon) .
\end{aligned}
$$

Using Lemma 3.4, for some constant $\gamma>0$, we have

$$
\begin{aligned}
|R(\epsilon)| & \leq \sum_{k=4}^{6} \int_{0}^{2 \pi} \int_{0}^{l} \epsilon\left\|\Delta \omega_{3 k}(x, u(x))\right\|_{L^{\infty}\left(\partial D_{\epsilon}\right)}\left|\frac{\partial u_{k-3}}{\partial \sigma}(\epsilon, \tilde{\theta}, \tau)\right| d \tau d \theta \\
& \leq \gamma \int_{0}^{2 \pi}\left\|\Delta \omega_{3 k}(x, u(x))\right\|_{L^{\infty}\left(\partial D_{\epsilon}\right)} d \theta \rightarrow 0,
\end{aligned}
$$

as $\epsilon \rightarrow 0$, by $(3.6)$ and being $\tilde{\omega}_{3 k}(\epsilon, \theta, \tau, u(\epsilon, \theta, \tau))$ uniformly continuous at $\epsilon=0$, again thanks to Lemma 3.10 and the fact that $C$ is compact. So that letting $\epsilon \rightarrow 0$ in (4.13), using Lemma 3.10, integrating by parts again, and taking into account the periodicity of $\omega$, (4.13) becomes

$$
\begin{aligned}
& -\sum_{k=4}^{6} \int_{0}^{l} \int_{0}^{2 \pi} \omega_{3 k}\left(\hat{\tau}, \frac{b_{1} \theta}{2 \pi}+c, \frac{b_{2} \theta}{2 \pi}+c, \frac{b_{3} \theta}{2 \pi}+c\right) \frac{b_{k-3}}{2 \pi} d \theta d \tau \\
& =-\frac{1}{2 \pi} \int_{0}^{l} \int_{0}^{2 \pi}\left\langle\omega\left(\hat{\tau}, \frac{b_{1} \theta}{2 \pi}, \frac{b_{2} \theta}{2 \pi}, \frac{b_{3} \theta}{2 \pi}\right), \bar{\tau} \wedge \bar{b}\right\rangle d \theta d \tau \\
& =\mathcal{L} \wedge \vec{b}(\omega),
\end{aligned}
$$

since in the local basis $\bar{\tau}=(\vec{\tau}, 0)=(0,0,1,0,0,0)$. The proof is completed.

4.2. Extensions of Theorem 4.1. In this section we discuss some extensions of Theorem 4.1. 


\subsubsection{The case with a finite number of loops.}

Theorem 4.5. Let $S$ be the union of $N>0$ Lipschitz surfaces $S_{k}$ in $\Omega$ whose boundary is $C$, the union of the corresponding boundaries $C_{k}$, that are closed curves in $\Omega$. Let $b=\left(b_{1}, b_{2}, b_{3}\right) \in 2 \pi \mathbb{Z}^{3}$ and let $u=\left(u_{1}, u_{2}, u_{3}\right): \Omega \rightarrow \mathbb{R}^{3}$ be a map with $u_{i}$ satisfying $(3.2)$ with $b=b_{i}$. Then $\mathcal{G}_{u}$ is an integral current in $\mathcal{D}_{3}\left(\Omega \times \mathbb{T}^{3}\right)$ and (4.4) holds.

Proof. Let us first suppose that $S_{k}$ and $C_{k}$ are smooth and that the curves $C_{k}$ are mutually disjoint. Then we will obtain the general result by approximation by means of Theorem 2.3 , arguing as in the proof of Corollary 4.3. Since $N$ is finite, we see that $C$ is compact and there is a tubular neighborhood around $C$. We can then argue as in the proof of Theorem 4.1, obtaining a formula similar to (4.13). Here $R(\epsilon)$ in (4.13) still vanishes thanks to Corollary 3.8, and $\frac{\partial u_{k-3}}{\partial \sigma}(\epsilon, \theta, \tau)$ in $(4.12)$ still tends to 0 . The thesis follows.

\subsubsection{Perturbation by a smooth function.}

Theorem 4.6. Let $S, C, b$ and $u$ as in Theorem 4.5, and let $v \in C^{1}\left(\bar{\Omega}, \mathbb{R}^{3}\right)$. Then $\mathcal{G}_{u+v}$ is the integral current in $\mathcal{D}_{3}\left(\Omega \times \mathbb{T}^{3}\right)$ given by

$$
\partial \mathcal{G}_{u+v}(\omega)=\mathcal{L} \wedge \vec{b}(\omega)+\mathcal{C}_{u+v}(\omega)
$$

for all $\omega \in \mathcal{D}^{2}\left(\Omega \times \mathbb{T}^{3}\right)$, with $\mathcal{C}_{u+v}$ defined as

$$
\mathcal{C}_{u+v}(\omega)=-\frac{1}{2 \pi} \int_{C} \int_{0}^{2 \pi}\left\langle\omega\left(x, \frac{b \theta}{2 \pi}+v(x)\right), \frac{\partial \bar{v}}{\partial \tau} \wedge \bar{b}\right\rangle d \theta d \mathcal{H}^{1}(x),
$$

for all $\omega \in \mathcal{D}^{2}\left(\Omega \times \mathbb{T}^{3}\right)$ and with $\bar{v}=(0, v) \in C^{1}\left(\bar{\Omega}, \mathbb{R}^{3} \times \mathbb{R}^{3}\right)$. In particular, it holds

$$
M\left(\partial \mathcal{G}_{u+v}\right) \leq C\left(1+\|D v\|_{L^{\infty}(\Omega)}\right)|\mathcal{L} \otimes b|(\Omega) .
$$

Proof. As in Theorem 4.1, we first prove the result for a smooth loop $C$ and then we obtain the general case arguing as in Theorem 4.5. Let us check that $u+v \in \mathcal{A}_{p}\left(\Omega, \mathbb{T}^{3}\right)$. To this aim let us prove that adj $(D u+D v)$ and $\operatorname{det}(D u+D v)$ are summable functions. Since the rows of $D u$ are linearly dependent and recalling the identity $\operatorname{det} A=\epsilon_{i j k} A_{1 i} A_{2 j} A_{3 k}=\epsilon_{i j k} A_{i 1} A_{j 2} A_{k 3}$, it follows that

$$
\operatorname{det}(D u+D v)=\operatorname{det}\left(\begin{array}{c}
D v_{1} \\
D v_{2} \\
D v_{3}
\end{array}\right)+\operatorname{det}\left(\begin{array}{c}
D u_{1} \\
D v_{2} \\
D v_{3}
\end{array}\right)+\operatorname{det}\left(\begin{array}{c}
D v_{1} \\
D u_{2} \\
D v_{3}
\end{array}\right)+\operatorname{det}\left(\begin{array}{c}
D v_{1} \\
D v_{2} \\
D u_{3}
\end{array}\right) .
$$

Since $D v_{i} \in C^{0}\left(\bar{\Omega}, \mathbb{R}^{3}\right)$, in particular it is bounded, so that all the determinants belong to $L^{p}\left(\Omega, \mathbb{R}^{3}\right)$ thanks to (3.6). A similar arguments applies for adj $(D u+D v)$.

To compute the boundary of $\mathcal{G}_{u+v}$ we proceed as in the proof of Corollary 4.3 (and Theorem 4.1 ), resulting in (4.10). This formula, setting $w:=u+v$, takes the form

$$
\begin{aligned}
& \int_{\partial D_{\epsilon}} \omega_{23}(x, w(x))-\sum_{k=1}^{3}\left(\omega_{2 k}(x, w(x)) \frac{\partial u_{k}}{\partial \tau}(x)+\omega_{3 k}(x, w(x)) \frac{\partial u_{k}}{\partial \sigma}(x)\right) d \mathcal{H}^{2}(x) \\
& -\int_{\partial D_{\epsilon}} \sum_{k=1}^{3}\left(\omega_{2 k}(x, w(x)) \frac{\partial v_{k}}{\partial \tau}(x)+\omega_{3 k}(x, w(x)) \frac{\partial v_{k}}{\partial \sigma}(x)\right) d \mathcal{H}^{2}(x)+ \\
& +\sum_{4 \leq i<j \leq 6} \int_{\partial D_{\epsilon}} \omega_{i j}(x, w(x)) \tilde{M}_{i}^{j}\left(D(\operatorname{Id} \times v)\left\llcorner\partial D_{\epsilon}(x)\right) d \mathcal{H}^{2}(x)\right. \\
& +\sum_{4 \leq i \neq j \leq 6} \int_{\partial D_{\epsilon}} \omega_{i j}(x, w(x))\left(\frac{\partial u_{i-3}}{\partial \tau} \frac{\partial v_{j-3}}{\partial \sigma}-\frac{\partial u_{i-3}}{\partial \sigma} \frac{\partial v_{j-3}}{\partial \tau}\right) d \mathcal{H}^{2}(x),
\end{aligned}
$$

where in the last term we have used the fact that $\omega_{i j}=-\omega_{j i}$. The first row, as seen by (4.11), tends to (4.14), the second and the third ones vanish as $\epsilon \rightarrow 0$ since $v$ is smooth and its partial derivatives 
are bounded. The terms of the last row containing $\frac{\partial u_{i-3}}{\partial \tau}$ and $\frac{\partial v_{j-3}}{\partial \sigma}$ vanish again thanks to Lemma 3.10 and the smoothness of $C$. It remains to study the term

$$
\begin{aligned}
& -\sum_{4 \leq i \neq j \leq 6} \int_{\partial D_{\epsilon}} \omega_{i j}(x, w(x)) \frac{\partial u_{i-3}}{\partial \sigma} \frac{\partial v_{j-3}}{\partial \tau} d \mathcal{H}^{2}(x)=R^{w}(\epsilon) \\
& -\sum_{4 \leq i \neq j \leq 6} \int_{0}^{l} \int_{0}^{2 \pi} \epsilon \tilde{\omega}_{i j}\left(\hat{\tau}, w\left(0^{+}, \theta, \tau\right)\right) \frac{\partial v_{j-3}}{\partial \tau}(\hat{\tau}) \frac{\partial u_{i-3}}{\partial \sigma}(\epsilon, \theta, \tau) d \theta d \tau,
\end{aligned}
$$

with $\hat{\tau}:=(0,0, \tau)$ and

$$
R^{w}(\epsilon)=-\sum_{4 \leq i \neq j \leq 6} \int_{0}^{l} \int_{0}^{2 \pi} \epsilon \Delta\left(\omega_{i j} \frac{\partial v_{j-3}}{\partial \tau}\right)(\epsilon, \theta, \tau) \frac{\partial u_{i-3}}{\partial \sigma}(\epsilon, \theta, \tau) d \theta d \tau
$$

and

$$
\Delta\left(\omega_{i j} \frac{\partial v_{j-3}}{\partial \tau}\right)(\epsilon, \theta, \tau):=\tilde{\omega}_{i j}(\epsilon, \theta, \tau, w(\epsilon, \theta, \tau)) \frac{\partial v_{j-3}}{\partial \tau}(\epsilon, \theta, \tau)-\tilde{\omega}_{i j}\left(\hat{\tau}, w\left(0^{+}, \theta, \tau\right)\right) \frac{\partial v_{j-3}}{\partial \tau}(\hat{\tau}) .
$$

Arguing as for $R(\epsilon)$ in (4.13) we see that $R^{w}(\epsilon)$ is negligible as $\epsilon \rightarrow 0$, while arguing as in (4.13) and taking into account that we see that $w\left(0^{+}, \theta, \tau\right)=\frac{b \theta}{2 \pi}+c+v(\hat{\tau})$, the expression (4.19) tends to

$$
\begin{aligned}
& -\sum_{4 \leq i \neq j \leq 6} \int_{0}^{l} \int_{0}^{2 \pi} \omega_{i j}\left(\hat{\tau}, \frac{b \theta}{2 \pi}+c+v(\hat{\tau})\right) \frac{\partial v_{j-3}}{\partial \tau}(\hat{\tau}) \frac{b_{i-3}}{2 \pi} d \theta d \tau \\
& =-\sum_{4 \leq i \neq j \leq 6} \int_{0}^{l} \int_{0}^{2 \pi} \omega_{i j}\left(\hat{\tau}, \frac{b \theta}{2 \pi}+v(\hat{\tau})\right) \frac{\partial v_{j-3}}{\partial \tau}(\hat{\tau}) \frac{b_{i-3}}{2 \pi} d \theta d \tau \\
& =-\sum_{4 \leq i<j \leq 6} \int_{0}^{l} \int_{0}^{2 \pi} \omega_{i j}\left(\hat{\tau}, \frac{b \theta}{2 \pi}+v(\hat{\tau})\right)\left(\frac{\partial v_{j-3}}{\partial \tau}(\hat{\tau}) \frac{b_{i-3}}{2 \pi}-\frac{\partial v_{i-3}}{\partial \tau}(\hat{\tau}) \frac{b_{j-3}}{2 \pi}\right) d \theta d \tau \\
& =\mathcal{C}_{u+v}(\omega),
\end{aligned}
$$

where we have considered the periodicity of $\omega$. The bound (4.17) now readily follows.

\section{Main Result SECOnd Form: EuClidean SETting AND Jump Surface}

In this section we express the boundary of the graph of $u$ as a current in $\Omega \times \mathbb{R}^{3}$, hence without neglecting the jump of $u$ on the surface $S$.

5.1. Expression of the boundary graph for $\mathbb{R}^{3}$-valued displacement. In this paper the main result Theorem 4.1 is presented without referring to the surface $S$ (in the final formulae), since torusvalued maps are considered. Nonetheless, the formulae in the Euclidean setting might be of interest with a view to the introduction of misfit energy terms as explained in Section 6.5. The derivation of such formulae is rather technical, though they do not require new notions with respect to those exposed in the paper core. This is the reason why the complete proofs are given in Appendix 5.

Let us introduce the following notation. For all $b \in \mathbb{R}^{3}$ and all $r \in \mathbb{R}^{3}$ we define the 1-current $\vec{b}_{r} \in \mathcal{D}_{1}\left(\mathbb{R}^{3}\right)$ as

$$
\vec{b}_{r}(\omega):=-\frac{1}{2 \pi} \int_{0}^{2 \pi}\left\langle\omega\left(r_{1}+\frac{b_{1} \theta}{2 \pi}, r_{2}+\frac{b_{2} \theta}{2 \pi}, r_{3}+\frac{b_{3} \theta}{2 \pi}\right), b\right\rangle d \theta
$$

for any 1-form $\omega \in \mathcal{D}^{1}\left(\mathbb{R}^{3}\right)$. Moreover $M\left(\vec{b}_{r}\right)=2 \pi|b|$ for all $r \in \mathbb{R}^{3}$.

Let $\tau$ be an arc length parameter along the curve $C$. As in Lemma 3.10 we will denote by $u(\tau)$ the value of the limit as $\epsilon \rightarrow 0$ of $u(\epsilon, \theta=0, \tau)$, with $\{\epsilon, \theta, \tau\}$ a system of cylindrical coordinates around $C$ taken in such a way that $\theta=0$ corresponds to the set of points in the surface $S$, so that the lower and upper traces of $u$ on $S$ can be denoted by $u(\epsilon, 0, \tau)$ and $u(\epsilon, 2 \pi, \tau)$, respectively (at 
least in a small neighborhood of $C$ ). Since the jump of $u$ is the constant $b$ on $S$ we can assume $u(\epsilon, 2 \pi, \tau)=b+u(\epsilon, 0, \tau)$. With this convention $u(\tau):=\lim _{\epsilon \rightarrow 0} u(\epsilon, 0, \tau)$.

We have denoted by $\mathcal{L} \wedge \vec{b}_{u(\tau)}$ the 2-current in $\Omega \times \mathbb{R}^{3}$ defined as

$$
\mathcal{L} \wedge \vec{b}_{u}(\omega)=-\frac{1}{2 \pi} \int_{C} \int_{0}^{2 \pi}\left\langle\omega\left(x, u(\tau)+\frac{b \theta}{2 \pi}\right), \bar{\tau} \wedge \bar{b}\right\rangle d \theta d \mathcal{H}^{1}(x),
$$

for any 2-form $\omega \in \mathcal{D}^{2}\left(\Omega \times \mathbb{R}^{3}\right)$.

Theorem 5.1. Let $C$ be a smooth closed curve in $\Omega$ and let $S$ be a smooth surface with $\partial S=C$. Let $b \in \mathbb{R}^{3}$ and let $u_{i} \in S B V(\Omega)$ be the solution to $(3.1)$ with $b=b_{i}$ for $i=1,2,3$. Let $v \in C^{1}\left(\bar{\Omega}, \mathbb{R}^{3}\right)$ and set $u=\left(u_{1}, u_{2}, u_{3}\right)$, and $w:=u+v$. Then $\mathcal{G}_{u}$ and $\mathcal{G}_{w}$ are integral currents in $\mathcal{D}_{3}\left(\Omega \times \mathbb{R}^{3}\right)$ and it holds

$$
\begin{aligned}
& \partial \mathcal{G}_{u}(\omega)=\mathcal{L} \wedge \vec{b}_{u}(\omega)+\int_{S}\left(\omega_{12}(x, u(x)+b)-\omega_{12}(x, u(x))\right) d \mathcal{H}^{2}(x) \\
& +\sum_{k=4}^{6} \int_{S} b_{k-3}\left(\omega_{1 k}(x, u(x)+b)-\omega_{1 k}(x, u(x))\right) \frac{\partial \hat{u}}{\partial \tau_{2}} d \mathcal{H}^{2}(x) \\
& -\sum_{k=4}^{6} \int_{S} b_{k-3}\left(\omega_{2 k}(x, u(x)+b)-\omega_{2 k}(x, u(x))\right) \frac{\partial \hat{u}}{\partial \tau_{1}} d \mathcal{H}^{2},
\end{aligned}
$$

for all $\omega \in \mathcal{D}^{2}\left(\Omega \times \mathbb{R}^{3}\right)$, where $\left\{\tau_{1}, \tau_{2}\right\}$ is an orthogonal basis for $S$, and $\hat{u}$ is the solution to (3.1) with $b=1$. In the previous formula $u$ and $u+b$ are the two traces of $u$ on the lower and upper face of $S$. Moreover

$$
\begin{aligned}
\partial \mathcal{G}_{w}(\omega)= & \sum_{4 \leq i<j \leq 6} \int_{S}\left(\omega_{i j}(x, w(x)+b)-\omega_{i j}(x, w(x))\right)\left(\frac{\partial w_{i-3}}{\partial \tau_{1}} \frac{\partial w_{j-3}}{\partial \tau_{2}}-\frac{\partial w_{j-3}}{\partial \tau_{1}} \frac{\partial w_{i-3}}{\partial \tau_{2}}\right) d \mathcal{H}^{2}(x) \\
& +\int_{S}\left(\omega_{12}(x, w(x)+b)-\omega_{12}(x, w(x))\right) d \mathcal{H}^{2}(x) \\
& +\sum_{k=4}^{6} \int_{S}\left(\omega_{1 k}(x, w(x)+b)-\omega_{1 k}(x, w(x))\right) \frac{\partial w_{k-3}}{\partial \tau_{2}} d \mathcal{H}^{2}(x) \\
& -\sum_{k=4}^{6} \int_{S}\left(\omega_{2 k}(x, w(x)+b)-\omega_{2 k}(x, w(x))\right) \frac{\partial w_{k-3}}{\partial \tau_{1}} d \mathcal{H}^{2}(x) \\
& +\mathcal{L} \wedge \vec{b}_{w}(\omega)+\mathcal{C}_{w}(\omega)
\end{aligned}
$$

for all $\omega \in \mathcal{D}^{2}\left(\Omega \times \mathbb{R}^{3}\right)$, where $\mathcal{C}_{w}(\omega)$ is a rectifiable 2 -current with support in $C \times \mathbb{R}^{3}$ given by

$$
\mathcal{C}_{w}(\omega):=-\frac{1}{2 \pi} \int_{C} \int_{0}^{2 \pi}\left\langle\omega\left(x, \frac{b \theta}{2 \pi}+w(x)\right), \frac{\partial \bar{v}}{\partial \tau}(x) \wedge \bar{b}\right\rangle d \theta d \mathcal{H}^{1}(x),
$$

with $\bar{v}=(0, v)$ and for all $\omega \in \mathcal{D}^{2}\left(\Omega \times \mathbb{R}^{3}\right)$. Moreover

$$
M\left(\mathcal{C}_{w}\right) \leq|\mathcal{L} \otimes b|(\Omega)\|D v\|_{\infty}
$$

In particular there exists a constant $c>0$ depending only on the geometry of $C$ such that

$$
M\left(\partial \mathcal{G}_{u}\right) \leq|\mathcal{L} \otimes b|(\Omega)+c \mathcal{H}^{2}(S)
$$

and

$$
M\left(\partial \mathcal{G}_{w}\right) \leq\left(1+\|D v\|_{\infty}\right)\left(|\mathcal{L} \otimes b|(\Omega)+c \mathcal{H}^{2}(S)\right)+\mathcal{H}^{2}(S)\|D v\|_{\infty}^{2} .
$$

For a proof we refer to Appendix B. 


\section{Application to Dislocations at the CONTINUUm SCALE}

6.1. Preliminaries on dislocations at the continuum scale. The following lemma characterizes the divergence-free measures which are supported on a 1-dimensional set as dislocation measures.

Lemma 6.1 (Dislocation measure). Let $\mu \in \mathcal{M}_{b}\left(\Omega, \mathbb{R}^{3 \times 3}\right)$ be a divergence-free measure which is absolutely continuous with respect to the $\mathcal{H}^{1}$-measure restricted on a simple Lipschitz curve $\mathcal{L}$ with tangent vector $\tau$ and such that $\mathcal{L}$ is either closed or ends at the boundary of $\Omega$. Then $\mu$ is a dislocationmeasure, that is, there exists a constant vector $b$ such that

$$
\mu=b \otimes \tau \mathcal{H}_{\llcorner\mathcal{L}}^{1} .
$$

Proof. By definition $\langle\mu, \varphi\rangle=\int_{\mathcal{L}} M(z) \cdot \varphi(z) d \mathcal{H}^{1}(z)$ for every $\varphi \in \mathcal{D}\left(\Omega, \mathbb{R}^{3 \times 3}\right)$, with $M \in L^{1}\left(\mathcal{L}, \mathbb{R}^{3 \times 3}\right)$. Moreover $\langle\mu, D \psi\rangle=0$ for every $\psi \in \mathcal{D}\left(\Omega, \mathbb{R}^{3}\right)$. Let $\{\nu, \sigma, \tau\}$ be a local orthogonal basis attached to $\mathcal{L}$. By orthogonal decomposition, $M_{i j}=M_{i k} \tau_{k} \tau_{j}+M_{i k} \nu_{k} \nu_{j}+M_{i k} \sigma_{k} \sigma_{j}$ and $\varphi_{i j}=\varphi_{i k} \tau_{k} \tau_{j}+\varphi_{i k} \nu_{k} \nu_{j}+$ $\varphi_{i k} \sigma_{k} \sigma_{j}$. It is easy to see that we can always choose $\varphi_{i j}=D_{j} \psi_{i}$ such that

$$
\partial_{\tau} \psi_{i \mid \mathcal{L}}=D_{j} \psi_{i} \tau_{j}=0, \quad \partial_{\nu} \psi_{i \mid \mathcal{L}}=\eta_{i}, \quad \partial_{\sigma} \psi_{i \mid \mathcal{L}}=\xi_{i},
$$

for arbitrary smooth functions $\eta$ and $\xi$ on $\mathcal{L}$, so that one has $0=\int_{\mathcal{L}} M(z) \cdot D \psi(z) d \mathcal{H}^{1}=\int_{\mathcal{L}}\left(M_{i j} \eta_{i} \nu_{j}\right.$ $\left.+\xi_{i} M_{i j} \sigma_{j}\right) d \mathcal{H}^{1}$, and hence $M_{i j}=b_{i} \tau_{j}$ with $b_{i}:=M_{i k} \tau_{k}$. Taking now $\varphi=D \psi$, it results from the closeness property of $\mathcal{L}$ and the compact support of $\psi$ that $0=\int_{\mathcal{L}} b \cdot \partial_{\tau} \psi_{\mid \mathcal{L}} d \mathcal{H}^{1}=-\int_{\mathcal{L}} \partial_{\tau} b \cdot \psi d \mathcal{H}^{1}$, and $b$ is constant.

According to Lemma 6.1, a dislocation loop is defined as a simple closed curve $\mathcal{L}$ in $\Omega$ which has an associated Burgers vector $b \in \mathbb{Z}^{3}$. The deformation gradient $F$ around $\mathcal{L}$ satisfies the condition

$$
-\operatorname{Curl} F=\Lambda_{\mathcal{L}^{b}}^{T}:=b \otimes \tau \mathcal{H}^{1}\llcorner\mathcal{L},
$$

where $\tau$ is an oriented tangent vector to $\mathcal{L}$. For any $b \in 2 \pi \mathbb{Z}^{3}$ we call a $b$-dislocation current a 1 -integer multiplicity current $\mathcal{L}^{b}$ that produces a curl of the deformation gradient given by the density $\Lambda_{\mathcal{L}^{b}}$, hence satisfying

$$
\left\langle\Lambda_{\mathcal{L}^{b}}, w\right\rangle=\mathcal{L}^{b}\left((w b)^{*}\right),
$$

for every $w \in \mathcal{C}_{c}^{\infty}\left(\Omega, \mathbb{R}^{3 \times 3}\right)$, where in the right-hand side $(w b)^{*}$ is the covector writing $(w b)^{*}:=$ $w_{k j} b_{j} d x_{k}$ (with sums on the repeated indexes). Moreover, $\Lambda_{\mathcal{L}^{b}}$ is a Radon measure as soon as $M\left(\mathcal{L}^{b}\right)$ is finite. In the sequel we will use the following shortcut notation:

$$
\Lambda_{\mathcal{L}^{b}}=\mathcal{L}^{b} \otimes b=\tau^{b} \otimes b \theta^{b} \mathcal{H}^{1}\left\llcorner_{\mathcal{L}}\right.
$$

with $\theta^{b}$ the multiplicity of the vector $b$ (see [29] for details).

Definition 6.2 (Regular dislocation). Let us set $\mathcal{B}:=2 \pi \mathbb{Z}^{3}$ the set of admissible Burgers vectors. A regular dislocation is a sequence of $b$-dislocation currents $\mathcal{L}:=\left\{\mathcal{L}^{b}\right\}_{b \in \mathcal{B}}$. We associate to each dislocation a dislocation current, still denoted by $\mathcal{L}$, and the associated dislocation density $\Lambda_{\mathcal{L}}$,

$$
\mathcal{L}:=\sum_{b \in \mathcal{B}} \mathcal{L}^{b}, \quad \Lambda_{\mathcal{L}}:=\sum_{b \in \mathcal{B}} \Lambda_{\mathcal{L}^{b}}
$$

With this definition it is possible to model the dislocations with every possible Burgers vector. It is possible to split the current $\mathcal{L}$ in the canonical basis of $\mathbb{R}^{3}$,

$$
\mathcal{L}=\mathcal{L}_{1}+\mathcal{L}_{2}+\mathcal{L}_{3},
$$

in such a way that $\mathcal{L}_{i}$ has $e_{i}$ as associated Burgers vector and satisfies

$$
\Lambda_{\mathcal{L}}=\Lambda_{\mathcal{L}_{1}}+\Lambda_{\mathcal{L}_{2}}+\Lambda_{\mathcal{L}_{3}}=\sum_{i=1}^{3} \mathcal{L}_{i} \otimes e_{i}
$$


Moreover, as proved in [29] one has

$$
\left|\mathcal{L}_{i}\right|_{\Omega} \leq C\left|\Lambda_{\mathcal{L}}\right|_{\mathcal{M}(\Omega)}
$$

for some constant $C$ independent of $i$ and $\Omega$.

6.2. Functional properties. Let $1 \leq p<\infty$ and introduce the vector space of tensor-valued fields

$$
\mathcal{B C}^{p}\left(\Omega, \mathbb{R}^{3 \times 3}\right):=\left\{F \in L^{p}\left(\Omega, \mathbb{R}^{3 \times 3}\right) \text { s.t. } \operatorname{Curl} F \in \mathcal{M}_{b}\left(\bar{\Omega}, \mathbb{R}^{3 \times 3}\right)\right\},
$$

which endowed with norm

$$
\|F\|_{\mathcal{B C}^{p}}:=\|F\|_{p}+|\operatorname{Curl} F|(\bar{\Omega}),
$$

is a Banach space. Let us define

$$
L_{\mathrm{div}}^{p}\left(\Omega, \mathbb{R}^{3 \times 3}\right):=\left\{F \in L^{p}(\Omega) \quad \text { s.t. } \quad \operatorname{div} F=0\right\},
$$

and the space

$$
\tilde{\mathcal{V}}^{p}(\Omega):=\left\{V \in L_{\text {div }}^{p}\left(\Omega, \mathbb{R}^{3 \times 3}\right) \text { s.t. } \operatorname{Curl} V \in L^{p}\left(\Omega, \mathbb{R}^{3 \times 3}\right), V N=0 \text { on } \partial \Omega\right\} .
$$

With a view to applications, it might be convenient to define another open set $\hat{\Omega}$ such that $\Omega \subset \subset \hat{\Omega}$. We will also assume that both $\Omega$ and $\hat{\Omega}$ are simply connected.

Let $1<p<2$ and let $F \in \mathcal{B C}^{p}\left(\hat{\Omega}, \mathbb{R}^{3 \times 3}\right)$ be such that, by virtue of Theorem 2.6 , - Curl $F=$ $b \otimes \mathcal{L}$, with $b \in 2 \pi \mathbb{Z}^{3}$ and $\mathcal{L}$ a 1-integer multiplicity current which is closed and with compact support in $\hat{\Omega}$. The Helmholtz decomposition in $L^{p}\left(\hat{\Omega}, \mathbb{R}^{3}\right)$ provides $v \in W^{1, p}\left(\hat{\Omega}, \mathbb{R}^{3}\right)$ and $G \in \tilde{\mathcal{V}}^{p}(\hat{\Omega})$ such that (see $[22]$ for details and further references)

$$
F=D v+\operatorname{Curl} G \text {. }
$$

If we set $V:=\operatorname{Curl} G$, then $\operatorname{div} V=0$, while since $-\operatorname{Curl} F=b \otimes \mathcal{L}$, we also have $-\operatorname{Curl} V=b \otimes \mathcal{L}$. Thanks to the decomposition theorem for 1-integer multiplicity currents (Theorem 2.1) we find a sequence of Lipschitz maps

$$
f_{k}: S^{1} \rightarrow \hat{\Omega} \quad \text { such that } \quad \mathcal{L}=\sum_{k>0} f_{k \sharp} \llbracket S^{1} \rrbracket .
$$

Let us denote by $\mathcal{L}_{k}$ the closed Lipschitz curves $f_{k}\left(S^{1}\right)$.

\subsection{Expression of Theorem 4.1 for a finite number of dislocation loops.}

Theorem 6.3. Let $b \in 2 \pi \mathbb{Z}^{3}$ be fixed, $1<p<2, \mathcal{L}$ be a closed integral current with compact support in $\hat{\Omega}$, and let $V \in \tilde{\mathcal{V}}^{p}(\hat{\Omega})$ be such that $-\operatorname{Curl} V=b \otimes \mathcal{L}$ in $\hat{\Omega}$. Then there exists a map $\tilde{u} \in \mathcal{A}_{p}\left(\hat{\Omega}, \mathbb{T}^{3}\right)$ such that $\nabla \tilde{u}=V$ almost everywhere in $\hat{\Omega}$, and

$$
M\left(\partial \mathcal{G}_{\tilde{u}}\right) \leq C|\mathcal{L} \otimes b|(\hat{\Omega})(1+|\mathcal{L} \otimes b|(\hat{\Omega})),
$$

with $C>0$ a constant depending only on $\hat{\Omega}$. Moreover $\tilde{u}=u-v$ with $v \in \mathcal{C}^{1}\left(\overline{\hat{\Omega}}, \mathbb{R}^{3}\right), u \in \mathcal{A}_{p}\left(\hat{\Omega}, \mathbb{T}^{3}\right)$, and

for all $\omega \in \mathcal{D}^{3}\left(\hat{\Omega} \times \mathbb{T}^{3}\right)$.

$$
\partial \mathcal{G}_{u}(\omega)=\mathcal{L} \wedge \vec{b}(\omega)=\Lambda_{\mathcal{L}}\left(\varphi^{\omega}\right):=\mathcal{L} \otimes b\left(\varphi^{\omega}\right)
$$

Recalling Remark 4.2, $\varphi^{\omega}$ is given by (4.6). Hence, Theorem 6.3 provides an expression of the dislocation density in terms of the boundary of the graph of the torus-valued displacement field $u$.

Proof. Let us first assume $p \leq \frac{3}{2}$. To prove the Theorem we will use the fact that the following system

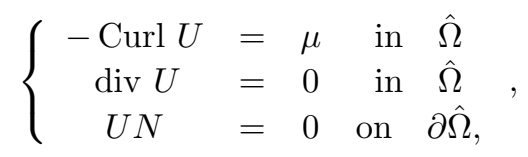


has by Theorem 2.6 a unique solution that also satisfies $\|U\|_{L^{p}} \leq C|\mu|(\hat{\Omega})$, with $C=C(\hat{\Omega})$. Another key fact is the following: if $\hat{\Omega}$ is a bounded open set with smooth boundary, $g \in \mathcal{C}^{0}\left(\partial \hat{\Omega}, \mathbb{R}^{3}\right)$ with $\int_{\partial \hat{\Omega}} g d \mathcal{H}^{2}=0$, and $v \in \mathcal{C}^{1}\left(\hat{\Omega}, \mathbb{R}^{3}\right)$ is the zero-mean-value solution to

$$
\left\{\begin{array}{cccc}
\Delta v & = & 0 & \text { in } \hat{\Omega} \\
\partial_{N} v & = & g & \text { on } \partial \hat{\Omega},
\end{array},\right.
$$

then $\|v\|_{\mathcal{C}^{1}} \leq C\|g\|_{\mathcal{C}^{0}}$, with $C=C(\hat{\Omega})$.

We use the decomposition (6.9) for $\mathcal{L}$ and we first suppose that the maps $f_{k}$ are smooth. The general case will follow using an approximation argument and proceeding as in the proof of Theorem 4.5. If $C_{k}$ is a smooth closed curve, we can choose a smooth surface $S_{k}$ with boundary $C_{k}$, according to Assumption 3.1. Then we set $S:=\cup_{k} S_{k}$ and $C:=\cup_{k} C_{k}$, we seek a solution $u$ of (3.1) with these $S$ and $C$. Let us also set $\hat{S}_{n}:=\cup_{k=0}^{n} S_{k}$ and $\hat{C}_{n}:=\cup_{k=0}^{n} C_{k}$. For $i=1,2,3$, let $u_{i}^{n}$ be the solution of (3.1) with $\hat{S}_{n}, \hat{C}_{n}$, and $b_{i}$. Lemma 3.13 and Remark 3.15 show that the distributional divergence of $\nabla u^{n}$ is zero, while the curl is given by $-b \otimes \sum_{k=0}^{n} f_{k \sharp} \llbracket S^{1} \rrbracket$. Up to subtracting a constant to $u^{n}$, we also suppose it has zero mean value.

By hypotheses it holds $\inf _{k} d\left(C_{k}, \partial \hat{\Omega}\right)>0$, and then $u^{n}$ are of class $\mathcal{C}^{\infty}$ on $\partial \hat{\Omega}$, and their $\mathcal{C}^{h}$ norms are uniformly bounded with respect to $n$ for all $h>0$ (taking into account that the set $C=\cup_{k} C_{k}$ has finite length, and then $S=\cup_{k} S_{k}$ has finite $\mathcal{H}^{2}$-measure). Let $v^{n}$ be the solution to (6.13) with $g:=\partial_{N} u^{n}$. From the estimates of this solution we find $\left\|v^{n}\right\|_{\mathcal{C}^{1}} \leq C_{1}\left\|\partial_{N} u^{n}\right\|_{\mathcal{C}^{0}}<C_{2}$, for some constant $C_{2}$ independent of $n$. Setting $\tilde{u}^{n}:=u^{n}-v^{n}$, we see that $\nabla \tilde{u}^{n}$ solves system (2.12) with $\mu=\mu^{n}:=b \otimes \sum_{k=0}^{n} f_{k \sharp} \llbracket S^{1} \rrbracket$, so that we also have $\left\|\nabla \tilde{u}^{n}\right\|_{p} \leq\left|\mu^{n}\right|(\hat{\Omega})<C_{3}$, with $C_{3}$ independent of $n$. In particular we get $\left\|u^{n}\right\|_{W^{1, p}} \leq\left\|v^{n}\right\|_{W^{1, p}}+\left\|\tilde{u}^{n}\right\|_{W^{1, p}} \leq C$, for a constant $C>0$ independent of $n$. Therefore, up to subsequences, $u^{n} \rightarrow u$ weakly in $W^{1, p}\left(\hat{\Omega}, \mathbb{R}^{3}\right)$, for some $u \in W^{1, p}\left(\hat{\Omega}, \mathbb{R}^{3}\right)$. Similarly $\tilde{u}^{n} \rightarrow \tilde{u}$ and $v^{n} \rightarrow v$ weakly in $W^{1, p}\left(\hat{\Omega}, \mathbb{R}^{3}\right)$, and from $u^{n}=\tilde{u}^{n}+v^{n}$ we infer $u=\tilde{u}+v$. Since the rows of $\nabla u^{n}$ are equal up to a multiplicative factor, we also get that all the minors of $u^{n}$ are uniformly bounded in $L^{p}$. Then, by Theorem 2.3 and Lemma 4.4, $u^{n}$ weakly converge in $\mathcal{A}_{p}\left(\hat{\Omega}, \mathbb{T}^{3}\right)$ to $u$ (up to extracting further subsequences). Moreover Theorem 4.5 implies that for every $n>0$ equation (4.4) holds for $u^{n}$, with $\mathcal{L}$ replaced by $\sum_{k=0}^{n} f_{k \sharp \sharp} \llbracket S^{1} \rrbracket$. Now, Lemma 2.5 implies that $\mathcal{G}_{u}$ is an integral current whose boundary satisfies

$$
\partial \mathcal{G}_{u}(\omega)=\sum_{k=1}^{\infty} f_{k \sharp} \llbracket S^{1} \rrbracket \wedge \vec{b}(\omega),
$$

for all $\omega \in \mathcal{D}^{3}\left(\hat{\Omega} \times \mathbb{T}^{3}\right)$. To conclude the proof it suffices to observe that the maps $\tilde{u}^{n}$ are smooth in a neighborhood of $\partial \hat{\Omega}$ with $\partial_{N} \tilde{u}^{n}$ vanishing, and hence $\partial_{N} \tilde{u}$ also vanishes, in such a way that $\nabla \tilde{u}$ satisfies (2.12) with $\mu:=b \otimes \mathcal{L}$. By the smoothness properties of $v^{n}$, it is also true that $v$ satisfies (6.13) with a bounded and smooth $g=\partial_{N} u$, so it is smooth in $\hat{\Omega}$ and Lemma 4.6 implies (4.17). We now compute $g$ by using formula (3.3) and $d\left(C_{k}, \partial \hat{\Omega}\right)>0$. The same argument employed in Lemma 3.4 (actually estimates (3.7) and (3.8)) shows that there is a constant $C_{4}>0$ such that $\|g\|_{\mathcal{C}^{1}} \leq C_{4}|b \otimes \mathcal{L}|(\hat{\Omega})$, so that the inequality $\|v\|_{\mathcal{C}^{1}} \leq C\|g\|_{\mathcal{C}^{0}}$ together with (4.17) gives (6.10). The thesis is proved when $p \leq \frac{3}{2}$. Assume $V \in L^{p}\left(\hat{\Omega}, \mathbb{R}^{3 \times 3}\right)$ with $\frac{3}{2}<p<2$. In particular $V \in L^{p}\left(\hat{\Omega}, \mathbb{R}^{3 \times 3}\right)$ with $p \leq \frac{3}{2}$, so the previous argument shows that $V=\nabla \tilde{u}=\nabla u-\nabla v$ with $\tilde{u}$ satisfying (6.10), $u$ satisfying (6.11), and $v$ of class $\mathcal{C}^{1}$. In particular $\nabla u \in L^{p}\left(\hat{\Omega}, \mathbb{R}^{3 \times 3}\right)$ with $\frac{3}{2}<p<2$, and the thesis follows.

Remark 6.4. By definition of the $u^{k}$, we have observed that for all $k$ the three components $u_{i}^{k}$, $i=1,2,3$, differ by a multiplicative factor. In particular we have seen that their gradients $\nabla u_{i}^{k}$ (i.e., the rows of the matrix $\nabla u^{k}$ ) are linearly dependent. As a consequence the same is true for the gradients $\nabla u_{i}$. Thus, the three components of the harmonic function $v$ have as boundary data $\partial_{N} u_{i}$ three linearly dependent vector fields. This implies, by the uniqueness of solution of elliptic equations, 
that also $\nabla v_{i}$ are linearly dependent and hence that the final matrix $V=\nabla u=\nabla \tilde{u}-\nabla v$ has linearly dependent rows. Therefore, its pointwise adjunct and determinant are constantly zero.

6.4. Two examples. In this section we show how Theorem 4.1 and its corollaries can be applied to obtain closure properties of suitable classes of deformations fields. This goal is achieved by means of two examples of variational problems. In both the examples we deal with two bounded, simply connected and smooth open sets $\Omega$ and $\hat{\Omega}$, with $\Omega \subset \subset \hat{\Omega}$. We fix a boundary datum $\alpha$ for the dislocation current in $\hat{\Omega} \backslash \Omega$ and a map $\hat{F} \in L^{p}\left(\hat{\Omega}, \mathbb{R}^{3 \times 3}\right)$ with $-\operatorname{Curl} \hat{F}=\Lambda_{\alpha}^{T}$ on $\hat{\Omega}$ and satisfying some suitable properties. Then we are interested in the minimization problem

$$
\min _{F \in \mathcal{F}} \mathcal{W}(F)
$$

where $F$ varies in a suitable class of admissible fields $\mathcal{F}$ satisfying $F=\hat{F}$ on $\tilde{\Omega} \backslash \Omega$.

6.4.1. A variational problem related to dislocations in finite elasticity. We deal with an energy $\mathcal{W}$ with the form

$$
\mathcal{W}(F):=\mathcal{W}_{e}(F)+\mathcal{W}_{\text {defect }}(\operatorname{Curl} F),
$$

where we assume the following properties on $\mathcal{W}_{e}$ and $\mathcal{W}_{\text {defect }}$ :

(i) The following coerciveness condition holds: there exists positive constants $\alpha_{0}, \alpha_{1}, \beta_{0}, \beta_{1}$, and $p, q \geq 1$ such that

$$
\begin{aligned}
& \mathcal{W}_{e}(F) \geq \beta_{1}\left(\|F\|_{L^{p}}^{p}+\|\operatorname{adj} F\|_{L^{p}}^{p}+\|\operatorname{det} F\|_{L^{p}}^{p}\right)-\beta_{0}, \\
& \mathcal{W}_{\text {defect }}(\Lambda) \geq \alpha_{1}|\Lambda|_{\mathcal{M}_{\mathrm{b}}}(\Omega)-\alpha_{0} .
\end{aligned}
$$

(ii) $\mathcal{W}_{\text {defect }}$ is a function on $\mathcal{M}_{b}\left(\Omega, \mathbb{R}^{3 \times 3}\right)$ which is lower semicontinuous with respect to the weak* convergence.

(iii) $\mathcal{W}_{e}$ is a function of $\mathcal{M}(F)$ (i.e., of $F$, adj $F$, and $\operatorname{det} F$ ), and it is lower semicontinuous in $\mathcal{M}(F)$ with respect to the weak convergence in $L^{p}$.

We assume that $\hat{F} \in \mathcal{A}^{p}\left(\hat{\Omega}, \mathbb{R}^{3 \times 3}\right)$ with $-\operatorname{Curl} \hat{F}=\Lambda_{\alpha}^{T}$ on $\hat{\Omega}$, and the additional property that $\operatorname{div} \hat{F}=0$ on $\hat{\Omega}$. Let $b \in 2 \pi \mathbb{Z}^{3}$ a fixed Burgers vector, let $p>1$, then the class of admissible deformation tensors is

$$
\begin{aligned}
& \mathcal{F}_{b}^{p}:=\left\{F \in L^{p}\left(\hat{\Omega}, \mathbb{R}^{3 \times 3}\right):-\operatorname{div} F=0,-\operatorname{Curl} F=b \otimes \mathcal{L}\right. \\
&\text { for some closed integral 1-current } \mathcal{L}, \text { and } F=\hat{F} \text { on } \hat{\Omega} \backslash \Omega\} .
\end{aligned}
$$

The minimization problem reads

$$
F \in \operatorname{argmin}_{F \in \mathcal{F}_{b}^{p}} \mathcal{W} .
$$

The existence of a solution is provided by the following:

Theorem 6.5. Let $1<p<2$. If $\mathcal{W}$ satisfies (i), (ii), and (iii), then there exists a solution $F \in \mathcal{F}_{b}^{p}$ of (6.19).

Proof. We will apply the direct method. Let $\left\{F_{k}\right\}_{k>0}$ be a minimizing sequence. From the coerciveness (i) we see that there exist $F \in L^{p}\left(\hat{\Omega}, \mathbb{R}^{3 \times 3}\right), A \in L^{p}\left(\hat{\Omega}, \mathbb{R}^{3 \times 3}\right)$, and $D \in L^{p}(\hat{\Omega})$, such that

$$
\begin{aligned}
& F_{k} \rightarrow F \quad \text { weakly in } L^{p}\left(\hat{\Omega}, \mathbb{R}^{3 \times 3}\right), \\
& \operatorname{adj} F_{k} \rightarrow A \quad \text { weakly in } L^{p}\left(\hat{\Omega}, \mathbb{R}^{3 \times 3}\right), \\
& \operatorname{det} F_{k} \rightarrow D \quad \text { weakly in } L^{p}(\hat{\Omega}),
\end{aligned}
$$

Moreover we find a measure $\Lambda \in \mathcal{M}_{b}\left(\hat{\Omega}, \mathbb{R}^{3 \times 3}\right)$ with

$$
\Lambda_{k} \rightarrow \Lambda \quad \text { weakly* in } \mathcal{M}_{b}\left(\hat{\Omega}, \mathbb{R}^{3 \times 3}\right),
$$


where we have set $\Lambda_{k}=\Lambda_{\mathcal{L}_{k}}=-\left(\operatorname{Curl} F_{k}\right)^{T}$. As [29, Lemma 7.5] shows, there exists a regular dislocation current $\mathcal{L}$ such that $\left(\mathcal{L}_{k}\right)_{i} \rightarrow \mathcal{L}_{i}$ in $\mathcal{D}_{1}(\hat{\Omega})$ and $\Lambda=\Lambda_{\mathcal{L}}=\sum_{i=1}^{3} \mathcal{L}_{i} \otimes e_{i}$. In order to prove the theorem we have to show that $\operatorname{div} F=0,-\operatorname{Curl} F=\Lambda_{\mathcal{L}}, A=\operatorname{adj} F$, and $D=\operatorname{det} F$.

The functions $F_{k}$ satisfy the hypotheses of Theorem 6.3, thus there exist a sequence of harmonic functions $\tilde{u}_{k} \in \mathcal{A}_{p}\left(\hat{\Omega}, \mathbb{T}^{3}\right)$ such that $\nabla \tilde{u}_{k}=F_{k}$ satisfying

$$
M\left(\partial \mathcal{G}_{\tilde{u}_{k}}\right) \leq C\left|\mathcal{L}_{k} \otimes b\right|(\hat{\Omega})\left(1+\left|\mathcal{L}_{k} \otimes b\right|(\hat{\Omega})\right) \leq M,
$$

for some constant $M>0$. By (6.20a) there exists $\tilde{u} \in \mathcal{A}_{p}\left(\hat{\Omega}, \mathbb{T}^{3}\right)$ such that, up to a subsequence,

$$
\tilde{u}_{k} \rightarrow \tilde{u} \text { weakly in } W^{1, p}\left(\hat{\Omega}, \mathbb{T}^{3}\right) \text {. }
$$

Now, thanks to (6.22), Theorem 2.3 applies, obtaining

$$
\begin{array}{ll}
\operatorname{adj}\left(\nabla \tilde{u}_{k}\right) \rightarrow \operatorname{adj}(\nabla \tilde{u}) & \text { weakly in } L^{p}\left(\hat{\Omega}, \mathbb{R}^{3 \times 3}\right), \\
\operatorname{det}\left(\nabla \tilde{u}_{k}\right) \rightarrow \operatorname{det}(\nabla \tilde{u}) & \text { weakly in } L^{p}(\hat{\Omega}) .
\end{array}
$$

These read $\operatorname{adj} F_{k} \rightarrow \operatorname{adj} F$ weakly in $L^{p}\left(\hat{\Omega}, \mathbb{R}^{3 \times 3}\right)$ and $\operatorname{det} F_{k} \rightarrow \operatorname{det} F$ weakly in $L^{p}\left(\hat{\Omega}, \mathbb{R}^{3}\right)$. Therefore $A=\operatorname{adj} F$ by $(6.20 \mathrm{~b})$, and $\operatorname{det} F=D$ from $(6.20 \mathrm{c})$. Moreover, for every test function $\varphi \in \mathcal{C}_{c}^{\infty}\left(\hat{\Omega}, \mathbb{R}^{3}\right)$ we have

$$
\langle F, \nabla \varphi\rangle=\langle\nabla w, \nabla \varphi\rangle=\lim _{k \rightarrow \infty}\left\langle\nabla w_{k}, \nabla \varphi\right\rangle=\lim _{k \rightarrow \infty}\left\langle\operatorname{div} F_{k}, \varphi\right\rangle=0,
$$

and it follows $\operatorname{div} F=0$ on $\hat{\Omega}$. Finally we write

$$
\left\langle\operatorname{Curl} F_{k}, \varphi\right\rangle=\left\langle F_{k}, \operatorname{Curl} \varphi\right\rangle \rightarrow\langle F, \operatorname{Curl} \varphi\rangle=\langle\operatorname{Curl} F, \varphi\rangle,
$$

for all $\varphi \in \mathcal{D}\left(\hat{\Omega}, \mathbb{R}^{3 \times 3}\right)$, and by $(6.21)$ we conclude $-\operatorname{Curl} F=\Lambda_{\mathcal{L}}^{T}$. The fact that $F$ satisfies the boundary condition is straightforward, and thus $F \in \mathcal{F}_{b}^{p}$, achieving the proof.

Remark 6.6. One cannot state that the above-solved variational problem is a complete solution to the dislocation problem, since what we have found in Theorem 6.5 is merely a divergence-free deformation, which is presumably not the deformation associated to the dislocation problem in finite elasticity. Indeed, this result must be considered as a partial result towards this aim. One idea is to exploit the decomposition $F=D u+\operatorname{Curl} V$ for the complete problem, and hence Theorem 6.5 provides only the divergence-free part Curl $V$ of the true deformation tensor $F$. Solving the complete problem is an extremely difficult task, without additional assumptions on $F$ or on the the nature of the dislocations. To this respect some results are found in [29].

6.4.2. A variational problem in finite gradient elasticity. In this example we treat a slightly different setting. We consider an energy involving $F$ and the gradient of $F$, viz. $\mathcal{W}=\tilde{\mathcal{W}}(F, \nabla F)$ where there are an energetic term for the curl of $F$ and another for its divergence. The term involving the divergence must be understood as a high-order perturbation, in the spirit of $[14]^{1}$, whereas the term related with the curl of $F$ will again be called the defect term, in view of the problem of the previous sections. Thus for some fixed $\epsilon>0$, we consider

$$
\tilde{\mathcal{W}}(F):=\mathcal{W}(F)+\epsilon \mathcal{W}_{\text {per }}(\operatorname{div} F) .
$$

The same assumptions (6.16) and (6.17) hold for $\mathcal{W}$, and for the perturbation, one assumes that

$$
\mathcal{W}_{\text {per }}(\operatorname{div} F) \geq \gamma\|\operatorname{div} F\|^{q},
$$

for $q>3$, where 3 is precisely the space dimension. Note that such an assumption could not be made on the whole gradient of $F$, since we know that Curl $F$ is a measure concentrated in the dislocations. Indeed, notice that, since - $\operatorname{Curl} F=\Lambda_{\mathcal{L}}^{T}$, the divergence of $F$ is not related to the dislocation density.

\footnotetext{
${ }^{1}$ In their case, since no dislocations were considered, $\operatorname{div} F=\Delta u$, with $u$ the displacement, was taken to the power 2 to account for the microstructure.
} 
We assume that the map $\hat{F} \in \mathcal{A}^{p}\left(\hat{\Omega}, \mathbb{R}^{3 \times 3}\right)$ with - Curl $\hat{F}=\Lambda_{\alpha}^{T}$ on $\hat{\Omega}$ satisfies the additional property that $\operatorname{div} \hat{F} \in L^{q}\left(\hat{\Omega}, \mathbb{R}^{3}\right)$. Let $b \in 2 \pi \mathbb{Z}^{3}$ a fixed Burgers vector, let $p, q>1$, then we define the class of admissible functions as

$$
\begin{aligned}
\mathcal{F}_{b}^{p, q}:= & \left\{F \in L^{p}\left(\hat{\Omega}, \mathbb{R}^{3 \times 3}\right):-\operatorname{div} F \in L^{q}\left(\hat{\Omega}, \mathbb{R}^{3}\right),-\operatorname{Curl} F=b \otimes \mathcal{L}\right. \\
& \text { for some closed integral 1-current } \mathcal{L}, \text { and } F=\hat{F} \text { on } \hat{\Omega} \backslash \Omega\} .
\end{aligned}
$$

The existence of a minimizer of $\mathcal{W}$ in $\mathcal{F}_{b}^{p, q}$ is provided by the following:

Theorem 6.7. Let $1<p<2$ and $q>3$. Assume that $\mathcal{W}$ satisfies the assumptions of Theorem 6.5. If $\mathcal{W}_{\text {per }}$ satisfies (6.27) and is lower semicontinuous with respect to the weak convergence in $L^{q}$, then there exists a minimizer $F \in \mathcal{F}_{b}^{p, q}$ of $\tilde{\mathcal{W}}$.

Proof. We will apply the direct method. Let $\left\{F_{k}\right\}_{k>0}$ be a minimizing sequence. From the coerciveness (i) we see that there exist $F \in L^{p}\left(\hat{\Omega}, \mathbb{R}^{3 \times 3}\right), A \in L^{p}\left(\hat{\Omega}, \mathbb{R}^{3 \times 3}\right), D \in L^{p}(\hat{\Omega})$, and $R \in L^{p}\left(\hat{\Omega}, \mathbb{R}^{3}\right)$ such that (6.20a)-(6.20c) hold, with moreover

$$
\operatorname{div} F_{k} \rightarrow R \quad \text { weakly in } L^{q}\left(\hat{\Omega}, \mathbb{R}^{3}\right) .
$$

We find a measure $\Lambda \in \mathcal{M}_{b}\left(\hat{\Omega}, \mathbb{R}^{3 \times 3}\right)$ with $\Lambda_{k} \rightarrow \Lambda$ weakly* in $\mathcal{M}_{b}\left(\hat{\Omega}, \mathbb{R}^{3 \times 3}\right)$, where we have set $\Lambda_{k}=\Lambda_{\mathcal{L}_{k}}=-\left(\operatorname{Curl} F_{k}\right)^{T}$. Again, [29, Lemma 7.5] implies that there exists a regular dislocation current $\mathcal{L}$ such that $\left(\mathcal{L}_{k}\right)_{i} \rightarrow \mathcal{L}_{i}$ in $\mathcal{D}_{1}(\hat{\Omega})$ and $\Lambda=\Lambda_{\mathcal{L}}=\sum_{i=1}^{3} \mathcal{L}_{i} \otimes e_{i}$. In order to prove the Theorem we have to show that $\operatorname{div} F=R,-\operatorname{Curl} F=\Lambda_{\mathcal{L}}, A=\operatorname{adj} F$, and $D=\operatorname{det} F$.

The Helmholtz decomposition gives

$$
F_{k}=D w_{k}+\operatorname{Curl} G_{k}
$$

with $w_{k} \in W^{1, p}\left(\hat{\Omega}, \mathbb{R}^{3}\right)$ satisfying $-\Delta w_{k}=-\operatorname{div} F_{k}$ with $\partial_{N} w_{k}=F_{k} N=\hat{F} N$ on $\partial \hat{\Omega}$ (note that $F_{k} N$ and $\hat{F} N$ have a distributional meaning, the divergences of $F_{k}$ and $\hat{F}$ being in $L^{p}$, see Lemma A.2), and $G_{k} \in \tilde{\mathcal{V}}^{p}(\hat{\Omega})$. Since $\operatorname{div} F_{k} \in L^{q}\left(\hat{\Omega}, \mathbb{R}^{3}\right)$, with $q>3$, by the regularity theory of elliptic problems and the Sobolev embedding Theorem, we find that $w_{k} \in \mathcal{C}^{1}\left(\hat{\Omega}, \mathbb{R}^{3 \times 3}\right)$ and that the $L^{\infty}$ norm of their gradients are bounded by a constant,

$$
\left\|D w_{k}\right\|_{\infty}<C .
$$

Moreover we have, up to a subsequence, that

$$
w_{k} \rightarrow w \quad \text { weakly in } W^{1, q}\left(\hat{\Omega}, \mathbb{R}^{3}\right),
$$

for some $w \in W^{1, q}\left(\hat{\Omega}, \mathbb{R}^{3}\right)$.

Let us set $V_{k}:=\operatorname{Curl} G_{k}$. Now $-\operatorname{Curl} V_{k}=\Lambda_{\mathcal{L}_{k}}^{T}$, and Theorem 6.3 provides functions $u_{k} \in \mathcal{A}_{p}\left(\hat{\Omega}, \mathbb{T}^{3}\right)$ and $v_{k} \in \mathcal{C}^{1}\left(\hat{\Omega}, \mathbb{T}^{3}\right)$ such that $\nabla u_{k}-\nabla v_{k}=V_{k}$ satisfying

$$
\partial \mathcal{G}_{u_{k}}(\omega)=\mathcal{L}_{k} \wedge \vec{b}(\omega),
$$

for all $\omega \in \mathcal{D}^{3}\left(\hat{\Omega} \times \mathbb{T}^{3}\right)$, and

$$
\left\|D v_{k}\right\|_{\infty} \leq C\left|b \otimes \mathcal{L}_{k}\right|(\hat{\Omega})
$$

Thanks to (6.20a), (6.32), and (6.34), we can assume that there exist $u \in W^{1, p}\left(\hat{\Omega}, \mathbb{T}^{3}\right)$ and $v \in$ $W^{1, p}\left(\hat{\Omega}, \mathbb{R}^{3 \times 3}\right)$ such that $u_{k} \rightarrow u$ and $v_{k} \rightarrow v$ strongly in $L^{p}\left(\hat{\Omega}, \mathbb{R}^{3}\right)$,

$$
\nabla u_{k} \rightarrow \nabla u \quad \text { weakly in } L^{p}\left(\hat{\Omega}, \mathbb{R}^{3 \times 3}\right),
$$

and

$$
\nabla v_{k} \rightarrow \nabla v \quad \text { weakly in } L^{p}\left(\hat{\Omega}, \mathbb{R}^{3 \times 3}\right) .
$$

Thanks to estimates (6.31) and (6.34), Theorem 4.6 applies providing

$$
M\left(\partial \mathcal{G}_{w_{k}+u_{k}-v_{k}}\right) \leq C\left(1+\left|\Lambda_{\mathcal{L}_{k}}\right|(\hat{\Omega})\right)\left|\Lambda_{\mathcal{L}_{k}}\right|(\hat{\Omega})<C .
$$

This allows us to apply Theorem 2.3, obtaining (6.24) and (6.25). Now, arguing as in Theorem 6.5 all other claims follow straightforwardly, achieving the proof. 
Remark 6.8. Once again, this is a partial solution to the complete dislocation problem. In particular, the growth assumption on the deformation divergence seems a relatively strong, which we would like to weaken in future works more oriented towards specific dislocation modelling.

6.5. Physical motivations of the Euclidean-valued case. With conclude with a quick remark on a possible reason in order to also consider vector-valued displacements in variational problems, without giving explicit examples.

Let us recall that the dislocated solid is separated into two elastic half-spaces joined by atomiclevel forces across their common interface, known as the glide plane. In the applications as shown so far, the energy is made of two terms, the elastic part and the defect part which is assumed as concentrated in $C$. With a view to time evolution of dislocation, an important physical term should also be added on the glide plane $S$, which is prescribed in single crystals at moderate temperature ranges, the so-called "misfit" energy taking into account nonlinear atomic interactions, viz.,

$$
\mathcal{W}_{\text {mis }}:=\int_{S} W_{\text {mis }}(\delta(x)) d S(x)
$$

where $0<\delta(x)<|b|$ and $W_{\text {mis }}$ is the interplanar potential energy density. This problem was originally introduced and solved by Nabarro, and received considerable attention nowadays, since it permits a reasonable alternative to atomic models.

\section{Appendix A. Proof of Theorem 2.6}

Here we prove Theorem 2.6. Let us first introduce some auxiliary results:

Lemma A.1. Let $G \in L^{p}\left(\Omega, \mathbb{R}^{3 \times 3}\right)$ with $1<p<\infty$ and $\Omega$ be a bounded open and simply-connected set with $C^{1}$ boundary. There exists a unique solution (up to a constant) $\phi \in W^{1, p}\left(\Omega, \mathbb{R}^{3}\right)$ of

$$
\left\{\begin{array}{l}
-\Delta \phi=\operatorname{div} G \quad \text { in } \Omega \\
\partial_{N} \phi=-G N \text { on } \partial \Omega
\end{array} .\right.
$$

Moreover such solution satisfies $\|D \phi\|_{p} \leq C\|G\|_{p}$. solved [34]:

Let us remark that equation (A.1) is a formal form meaning that the following weak form is

$$
-\langle\nabla \phi, \nabla \varphi\rangle=\langle G, \nabla \varphi\rangle \quad \forall \varphi \in W^{1, p^{\prime}}\left(\Omega, \mathbb{R}^{3 \times 3}\right) .
$$

Proof. This Lemma is a direct tensor extension of the theorems of existence and uniqueness of Neumann problem as shown in [31] (see also [15, Lemma III.1.2 and Theorem III.1.2]).

The following result can be proven (see [32]):

Lemma A.2. Let $\Omega \subset \mathbb{R}^{3}$ be a bounded open set with boundary of class $C^{1}$ and let $F \in L^{p}\left(\Omega, \mathbb{R}^{3 \times 3}\right)$ be such that $\operatorname{div} F \in L^{p}\left(\Omega, \mathbb{R}^{3}\right)$. Then the distribution defined as

$$
\langle F N, \gamma(\varphi)\rangle:=\langle\operatorname{div} F, \varphi\rangle+\langle F, D \varphi\rangle
$$

for all $\varphi \in W^{1, p^{\prime}}\left(\Omega, \mathbb{R}^{3}\right)$, with $\gamma(\varphi) \in W^{1 / p, p^{\prime}}\left(\partial \Omega, \mathbb{R}^{3}\right)$ the boundary trace of $\varphi$, is well-defined and it holds $F N \in W^{-1 / p, p}\left(\partial \Omega, \mathbb{R}^{3}\right):=\left(W^{1 / p, p^{\prime}}\left(\partial \Omega, \mathbb{R}^{3}\right)\right)^{\prime}$.

We are ready to prove Theorem 2.6 .

Proof. Let us discuss the proof in several steps. In the sequel $\Phi$ represents the fundamental solution of the Laplacian in $\mathbb{R}^{3}$, i.e., $\Delta \Phi=\delta_{0}$.

Step 1: Existence and explicit expression. 
Employing Theorem 2 and 3 of [8] we get the existence of a solution to

$$
\left\{\begin{array}{ccccc}
-\operatorname{Curl} G & = & \text { in } & \hat{\Omega} \\
\operatorname{div} G & = & 0 & \text { in } \hat{\Omega}
\end{array}\right.
$$

whenever $\mu \in L^{1}\left(\hat{\Omega}, \mathbb{R}^{3 \times 3}\right)$. The general case $\mu \in \mathcal{M}_{\text {div }}\left(\hat{\Omega}, \mathbb{R}^{3 \times 3}\right)$ is thus obtained by approximation. Moreover it is seen that the explicit solution $G$ of (A.4) is componentwise

$$
G_{j m}(x):=-\epsilon_{m l k}\left(D_{l} \Phi \star \mu_{k j}\right)(x)=-\int_{\mathbb{R}^{3}} \epsilon_{m l k} D_{l} \Phi(\xi-x) d \mu_{k j}(\xi) .
$$

Step 2: boundedness.

The continuity property $(2.13)$ on $L^{p}\left(\mathbb{R}^{3}, \mathbb{R}^{3 \times 3}\right)$ with $1 \leq p<3 / 2$ follows from $[8$, Theorem 2 and Theorem 3] and approximation. But it can also be obtained directly first observing that $D \Phi \in$ $L^{p}\left(\mathbb{R}^{3}, \mathbb{R}^{3 \times 3}\right)$ with $1 \leq p<3 / 2$, since $D \Phi(x)=O\left(|x|^{-2}\right)$, and hence, posing $R:=|x-y|$,

$$
\|D \Phi\|_{p}^{p} \leq \int_{0}^{\bar{R}} R^{-2 p} R^{2} d R
$$

where $\bar{R}$ is the radius of a ball centered in 0 and containing $\hat{\Omega}$. It is then observed that the last factor in the right-hand side is bounded as long as $1 \leq p<3 / 2$. The boundedness in $L^{p}$ (hence the continuity) now follows from Minkowski's inequality since for some $C>0$, it holds (see also [30, VI.I;4]),

$$
\|G\|_{p} \leq C|\mu|(\hat{\Omega}) .
$$

Step 3: vanishing of $F N$.

By Lemma A.2, since $\operatorname{div} G=0$ the normal trace of $G$, denoted as $G N$, exists as an element of $W^{-1 / p, p}\left(\partial \hat{\Omega}, \mathbb{R}^{3}\right)$ and satisfies

$$
\langle G N, \varphi\rangle_{\partial \hat{\Omega}}:=\langle G, \nabla \varphi\rangle+\langle\operatorname{div} G, \varphi\rangle=\langle G, \nabla \varphi\rangle \quad \forall \varphi \in W^{1, p^{\prime}}\left(\hat{\Omega}, \mathbb{R}^{3}\right) .
$$

If $\phi \in W^{1, p}(\hat{\Omega})$ we have that $F:=G+D \phi$ also satisfies - Curl $F=\mu^{\mathrm{T}}$. Lemma A.1 provides a solution $\phi$ such that $\operatorname{div} F=0$ in $\hat{\Omega}, F N=0$ on $\partial \hat{\Omega}$, and such that (2.13) holds.

Step 4: uniqueness.

Assume that there exist two solutions and denote by $H \in L^{p}(\hat{\Omega})$ their difference, one has Curl $H=$ $\operatorname{div} H=0$ in $\hat{\Omega}$ while $H N=0$ on $\partial \hat{\Omega}$. There exists $u \in W^{1, p}\left(\hat{\Omega}, \mathbb{R}^{3}\right)$ such that $H=\nabla u$. Taking the divergence one gets $\Delta u=0$ in $\hat{\Omega}$. Moreover from $H N=0$ we also have $\partial_{N} u=0$ on $\partial \hat{\Omega}$. By Lemma A.1 this implies that there is a constant $c$ with $u \equiv c$ in $\hat{\Omega}$, whereby $H=0$.

Step 5: sharp result for a smooth curve.

Let $\mu^{T}$ be a concentrated measure on a smooth curve $L \subset \hat{\Omega}$, i.e., $\mu^{T}=M \cdot \mathcal{H}_{L}^{1}$, and $M_{i j}(z)=b_{i} \tau_{j}(z)$ with constant $b_{i}$ (by Lemma 6.1). Since $L$ has bounded curvature there exists $\epsilon>0$ and a constant $c>0$ such that there holds

$$
\forall \delta<\epsilon \quad \mathcal{H}^{2}(\{x: d(x, L)=\delta\}) \leq 2 \pi l c \delta
$$

where $l$ is the length of the curve $L$. Formula (A.5) implies

$$
G_{j l}(x)=-\epsilon_{l k i} D_{k} \Phi \star \mu_{i j}^{T}=-\int_{L} b_{i} \epsilon_{l k i} D_{k} \Phi(\xi-x) \tau_{j}(\xi) d \mathcal{H}^{1}(\xi) .
$$


Now $D \Phi \sim \frac{1}{x^{2}}$, and then $D \Phi \star \mu^{T} \sim \frac{1}{d^{2}(x, L)}$. Using (A.8) and employing the Coarea formula we estimate

$$
\begin{aligned}
& \int_{\hat{\Omega}}\|G\|^{p} d x \leq \int_{\hat{\Omega}}\|G\|^{p-1}\left\|D\left(\Phi \star \mu^{T}\right)\right\| d x=\int_{0}^{\bar{R}} \int_{\left\{\Phi \star \mu^{T}=r\right\}}\|G\|^{p-1} d r \\
& \sim \int_{0}^{\bar{R}} 2 \pi l c \frac{r}{\left(r^{2}\right)^{p-1}} d r<+\infty
\end{aligned}
$$

whenever $p<2$. The proof is achieved.

\section{Appendix B. Proof of Theorem 5.1}

Proof. We will follow the lines of the proof of Theorem 4.1. We consider a smooth tubular neighborhood $D_{\epsilon}$ of the curve $C$, and a neighborhood $U_{\delta}$, of widht $2 \delta$, of the surface $S$. Then we consider the currents $\partial \mathcal{G}_{u^{\epsilon, \delta}}$ in $\Omega \times \mathbb{R}^{3}$, with $u^{\epsilon, \delta}:=u\left\llcorner\Omega \backslash\left(D_{\epsilon} \cup U_{\delta}\right)\right.$ and let first $\delta \rightarrow 0$, and then $\epsilon \rightarrow 0$.

To simplify the notation, we denote by $\partial D_{\epsilon}$ only the part of the boundary of $D_{\epsilon}$ that does not belong to $U_{\delta}$, and similarly $\partial U_{\delta}$ is the boundary of $U_{\delta}$ which does not belong to $D_{\epsilon}$. Therefore, for all $\omega \in \mathcal{D}^{2}\left(\Omega \times \mathbb{R}^{3}\right)$ it holds

$$
\begin{aligned}
\partial \mathcal{G}_{u^{\epsilon, \delta}}(\omega)= & \int_{\partial D_{\epsilon}} \sigma(i, \bar{i}) \omega_{i j}(x, u(x)) \tilde{M}_{i}^{j}\left(D\left(\Phi\left\llcorner\partial D_{\epsilon}(x)\right)\right) d \mathcal{H}^{2}(x)\right. \\
& +\int_{\partial U_{\delta}} \sigma(i, \bar{i}) \omega_{i j}(x, u(x)) \tilde{M}_{i}^{j}\left(D\left(\Phi\left\llcorner\partial U_{\delta}(x)\right)\right) d \mathcal{H}^{2},\right.
\end{aligned}
$$

where $\Phi:=I d \times u$. Let us study the second term of the last expression. Using the coordinates $\left\{\tau_{1}, \tau_{2}\right\}$ on $S$ and denoting by $N$ the unit normal to $S$, this can be written as

$$
\begin{aligned}
& \int_{\partial S} \omega_{12}(x+\delta N, u(x+\delta N))+\sum_{k=4}^{6} \omega_{1 k}(x+\delta N, u(x+\delta N)) \frac{\partial u_{k-3}}{\partial \tau_{2}}(x+\delta N) d \mathcal{H}^{2}(x) \\
& -\int_{\partial S} \sum_{k=4}^{6} \omega_{2 k}(x+\delta N, u(x+\delta N)) \frac{\partial u_{k-3}}{\partial \tau_{1}}(x+\delta N) d \mathcal{H}^{2}(x) \\
& -\int_{\partial S} \omega_{12}(x-\delta N, u(x-\delta N))-\sum_{k=4}^{6} \omega_{1 k}(x-\delta N, u(x-\delta N)) \frac{\partial u_{k-3}}{\partial \tau_{2}}(x-\delta N) d \mathcal{H}^{2}(x) \\
& +\int_{\partial S} \sum_{k=4}^{6} \omega_{2 k}(x-\delta N, u(x-\delta N)) \frac{\partial u_{k-3}}{\partial \tau_{1}}(x-\delta N) d \mathcal{H}^{2}(x) .
\end{aligned}
$$

Using the fact that $d\left(\partial S_{\delta}, C\right) \geq \epsilon$, we see that such a current, thanks to (3.6), has uniformly bounded mass for all $\delta$, so that the currents $\mathcal{G}_{u^{\epsilon, \delta}}$ converge to $\mathcal{G}_{u^{\epsilon, \delta}=0}$ in the sense of currents. Letting $\delta \rightarrow 0$ in (B.2), taking into account that $u_{k}=b_{k} \hat{u}$, we obtain exactly the second, third, and fourth terms in (5.3), with the only exception that we are integrating on $S \backslash D_{\epsilon}$, viz.,

$$
\begin{aligned}
\partial \mathcal{G}_{u^{\epsilon}, 0}(\omega)= & \int_{\partial D_{\epsilon}} \sigma(i, \bar{i}) \omega_{i j}(x, u(x)) \tilde{M}_{i}^{j}\left(D\left(\Phi\left\llcorner\partial D_{\epsilon}(x)\right)\right) d \mathcal{H}^{2}(x)\right. \\
& +\int_{S \backslash D_{\epsilon}}\left(\omega_{12}(x, u(x)+b)-\omega_{12}(x, u(x))\right) d \mathcal{H}^{2}(x) \\
& +\sum_{k=4}^{6} \int_{S \backslash D_{\epsilon}} b_{k-3}\left(\omega_{1 k}(x, u(x)+b)-\omega_{1 k}(x, u(x))\right) \frac{\partial \hat{u}}{\partial \tau_{2}} d \mathcal{H}^{2}(x) \\
& -\sum_{k=4}^{6} \int_{S \backslash D_{\epsilon}} b_{k-3}\left(\omega_{2 k}(x, u(x)+b)-\omega_{2 k}(x, u(x))\right) \frac{\partial \hat{u}}{\partial \tau_{1}} d \mathcal{H}^{2}(x) .
\end{aligned}
$$


Now, we can explicitly write the first integral as in (4.11), i.e.,

$$
\begin{aligned}
& \int_{\partial D_{\epsilon}} \tilde{\omega}_{23}(x, u(x)) d \mathcal{H}^{2}-\sum_{k=4}^{6} \int_{0}^{2 \pi} \int_{0}^{l} \epsilon \tilde{\omega}_{2 k}(\epsilon, \theta, \tau, u(\epsilon, \theta, \tau)) \frac{\partial u_{k-3}}{\partial \tau}(\epsilon, \theta, \tau) d \tau d \theta \\
& \left.-\sum_{k=4}^{6} \int_{0}^{l} \int_{0}^{2 \pi} \epsilon \tilde{\omega}_{3 k}(\epsilon, \theta, \tau, u(\epsilon, \theta, \tau)) \frac{\partial u_{k-3}}{\partial \sigma}(\epsilon, \theta, \tau)\right) d \theta d \tau,
\end{aligned}
$$

and following the same computations as in the proof of Theorem 4.1, neglecting the vanishing terms, we obtain the following expression

$$
\begin{aligned}
& -\left.\sum_{k=4}^{6} \int_{0}^{l} \omega_{3 k}\left(\hat{\tau}, u^{+}(\tau)\right) u_{k-3}(\epsilon, \theta, \tau)\right|_{0} ^{\theta=2 \pi} d \tau \\
& \left.+\sum_{k=4}^{6} \int_{0}^{l} \int_{0}^{2 \pi} \frac{d}{d \theta} \omega_{3 k}\left(\hat{\tau}, u^{+}(\tau)\right) u_{k-3}(\epsilon, \theta, \tau)\right) d \theta d \tau
\end{aligned}
$$

with $\hat{\tau}=(0,0, \tau)$. Letting $\epsilon$ go to 0 and integrating by parts in $\theta$ as in (4.14), we obtain

$$
-\sum_{k=4}^{6} \int_{0}^{l} \int_{0}^{2 \pi} \omega_{3 k}\left(\hat{\tau}, u^{+}(\tau)+\frac{b}{2 \pi} \theta\right) \frac{b_{k-3}}{2 \pi} d \theta d \tau
$$

where we have used that $\lim _{\epsilon \rightarrow 0} u(\epsilon, \theta, \tau)=u^{+}(\tau)=u^{+}(\tau)+\frac{b \theta}{2 \pi}$. This last expression is exactly the first term of (5.3). To see that the second, third, and fourth terms of (B.3) tend to the correspondent terms in (5.3), it suffices to observe that the total mass of the currents represented by them are bounded (uniformly with respect to $\epsilon$ ) thanks to the estimates (ii) and (iii) of Lemma 3.10, so that $\mathcal{G}_{u^{\epsilon, 0}}$ converges to $\mathcal{G}_{u}$ in the sense of currents and we are done.

It remains to prove (5.4). We argue as in the proof of Theorem 4.6. The previous computations applied to the function $w$ gives rise to a formula like (B.3) for the part of the boundary on $S$ plus the term containing the $2 \times 2$ determinants of $D w$. These terms converge to the first four lines of (5.4). As for the part of the boundary on $C$ we have an approximation as in (4.18), where the first row can be treated as in (B.4). This gives rise to $\mathcal{L} \wedge \vec{b}_{w}(\omega)$. The other non-vanishing terms are given by the expression (4.19), with the same notation. In contrast we have that $\omega$ is not anymore periodic. Then arguing as in (4.19) we find the final expression

$$
-\sum_{4 \leq i \neq j \leq 6} \int_{0}^{l} \int_{0}^{2 \pi} \omega_{i j}\left(\hat{\tau}, \frac{b \theta}{2 \pi}+w(\hat{\tau})\right) \frac{\partial v_{j-3}}{\partial \tau}(\hat{\tau}) \frac{b_{i-3}}{2 \pi} d \theta d \tau=\mathcal{C}_{w}(\omega),
$$

with $\hat{\tau}=(0,0, \tau)$ as expressed in the local basis. Now $\mathcal{C}_{w}$ can be estimated as in Theorem 4.6. The estimates (5.6) and (5.7) are straightforward consequences of (5.3) and (5.4).

Acknowledgements. The financial support of the FP7-IDEAS-ERC-StG \#256872 (EntroPhase) is gratefully acknowledged by the first author. The present paper also benefits from the support of the GNAMPA (Gruppo Nazionale per l'Analisi Matematica, la Probabilità e le loro Applicazioni) of INdAM (Istituto Nazionale di Alta Matematica). Second author was supported by the FCT Starting Grant " Mathematical theory of dislocations : geometry, analysis, and modelling" (IF/00734/2013).

\section{REFERENCES}

[1] A. Aftalion, E. Sandier, and S. Serfaty. Pinning phenomena in the Ginzburg-Landau model of superconductivity. J. Math. Pures Appl. (9), 80(3):339-372, 2001.

[2] G. Alberti. On maps whose distributional Jacobian is a measure. Real Anal. Exch., 33:153-162, 2008.

[3] G. Alberti, S. Baldo, and G. Orlandi. Functions with prescribed singularities. J. Eur. Math. Soc. (JEMS), 5(3):275$311,2003$.

[4] F. Almgren. Deformations and multiple-valued functions. Geometric measure theory and the calculus of variations, Proc. Summer Inst., Arcata/Calif. 1984, Proc. Symp. Pure Math. 44, 29-30 (1986)., 1986. 
[5] J. M. Ball. Convexity conditions and existence theorems in nonlinear elasticity. Arch. Ration. Mech. Anal., 63:337403, 1977.

[6] F. Bethuel, G. Orlandi, and D. Smets. Motion of concentration sets in Ginzburg-Landau equations. Ann. Fac. Sci. Toulouse, Math. (6), 13(1):3-43, 2004.

[7] Ph. Bouafia, T.De Pauw, and C. Wang. Multiple valued maps into a separable Hilbert space that almost minimize their $p$ Dirichlet energy or are squeeze and squash stationary. Calc. Var. Partial Differ. Equ., 54(2):2167-2196, 2015.

[8] J. Bourgain and A. Brezis. New estimates for the Laplacian, the divcurl, and related Hodge systems. Comptes Rendus Matematique, 338(I):539543, 2004.

[9] Y. Brenier. Non relativistic strings may be approximated by relativistic strings. Methods Appl. Anal., 12(2):153-167, 2005.

[10] H. Brézis, J.-M. Coron, and E. H. Lieb. Harmonic maps with defects. Commun. Math. Phys., 107:649-705, 1986.

[11] P.G. Ciarlet. Three-Dimensional Elasticity. Mathematical Elasticity: Three-dimensional Elasticity. North-Holland, 1994.

[12] C. De Lellis, C.-R. Grisanti, and P. Tilli. Regular selections for multiple-valued functions. Ann. Mat. Pura Appl. (4), 183(1):79-95, 2004.

[13] H. Federer. Geometric measure theory. Springer-Verlag, Berlin, Heidelberg, New York., 1969.

[14] G. A. Francfort and S. Müller. Combined effects of homogenization and singular perturbations in elasticity. J. Reine Angew. Math., 454:1-35, 1994.

[15] G. P. Galdi. An introduction to the mathematical theory of the Navier-Stokes equations. Steady-state problems. 2nd ed. New York, NY: Springer., 2011.

[16] M. Giaquinta, P. M. Mariano, G. Modica, and D. Mucci. Currents and curvature varifolds in continuum mechanics. In Nonlinear partial differential equations and related topics. Dedicated to Nina U. Uraltseva on the occasion of her 75th birthday., pages 97-117. Providence, RI: American Mathematical Society (AMS), 2010.

[17] M. Giaquinta, G. Modica, and J. Souček. Cartesian currents in the calculus of variations I. Cartesian currents. Ergebnisse der Mathematik und ihrer Grenzgebiete. 3. Folge. 37. Berlin: Springer, 1998.

[18] M. Giaquinta, G. Modica, and J. Souček. Cartesian currents in the calculus of variations II. Variational integrals. Ergebnisse der Mathematik und ihrer Grenzgebiete. 3. Folge. 38. Berlin: Springer, 1998.

[19] D. Henao and S. Serfaty. Energy estimates and cavity interaction for a critical-exponent cavitation model. Commun. Pure Appl. Math., 66(7):1028-1101, 2013.

[20] R. L. Jerrard and H. M. Soner. Functions of bounded higher variation. Indiana Univ. Math. J., 51:645677, 2002.

[21] H. Kleinert. Gauge fields in condensed matter, Vol.1. World Scientific Publishing, Singapore, 1989.

[22] H. Kozono and T. Yanagisawa. $L^{r}$-variational inequality for vector fields and the Helmholtz-Weyl decomposition in bounded domains. Indiana Univ. Math. J., 58(4):1853-1920, 2009.

[23] S.G. Krantz and H.R. Parks. Geometric Integration Theory. Cornerstones. Birkhäuser, 2008.

[24] D. Mucci. Fractures and vector valued maps. Calc. Var. Partial Differ. Equ., 22(4):391-420, 2005.

[25] D. Mucci. A structure property of "vertical" integral currents, with an application to the distributional determinant. Rev. Mat. Complut., 28(1):49-83, 2015.

[26] S. Müller. Det=det. A remark on the distributional determinant. C. R. Acad. Sci., Paris, Sér. I 311, 1:13-17, 1990.

[27] S. Müller and M. Palombaro. Existence of minimizers for a polyconvex energy in a crystal with dislocations. Calc. Var., 31(4):473-482, 2008.

[28] E. Sandier and S. Serfaty. A product-estimate for Ginzburg-Landau and corollaries. J. Funct. Anal., 211(1):219244, 2004

[29] R. Scala and N. Van Goethem. Currents and dislocations at the continuum scale. Methods Appl. Anal., 23(1):1-34, 2016.

[30] L. Schwartz. Théorie des distributions. Hermann, Paris, 1957.

[31] C. G. Simader and H. Sohr. The Dirichlet problem for the Laplacian in bounded and unbounded domains. Pitman Research Notes in Mathematics Series. 360. Harlow: Addison Wesley Longman. , 1996.

[32] N. Van Goethem. Cauchy elasticity with dislocations in the small strain assumption. Appl. Math. Lett., 46:94-99, 2015 .

[33] N. Van Goethem. Incompatibility-governed singularities in linear elasticity with dislocations. Math. Mech. Solids, (to appear)(https://hal.archives-ouvertes.fr (\# hal-01203034)), 2016

[34] T. Yanagisawa. Hodge decomposition of $L^{r}$-vector fields on a bounded domain and its application to the NavierStokes equations. Conference Proceedings RIMS (Kyoto Univ.), 1536:73-86, 2007. 
Weierstrass Institute for Applied Analysis and Stochastics Germany

E-mail address: riccardo.scala@wias-berlin.de

Universidade de Lisboa, Faculdade de Ciências, Departamento de Matemática, CMAF, Av. Prof. Gama Pinto 2, 1649-003 Lisboa, Portugal

E-mail address: vangoeth@fc.ul.pt 